\title{
REVISED Evolution of human respiratory virus epidemics [version
}

\section{2; peer review: 2 approved]}

\author{
Nash Rochman (D), Yuri I Wolf, Eugene V Koonin (iD) \\ National Center for Biotechnology Information, National Institutes of Health, Bethesda, MD, 20894, USA
}

V2 First published: 04 Jun 2021, 10:447

https://doi.org/10.12688/f1000research.53392.1

Latest published: $29 \mathrm{Jul}$ 2021, 10:447

https://doi.org/10.12688/f1000research.53392.2

\section{Abstract}

Background: Pathogens are often assumed to evolve towards reduced virulence, but counterexamples abound. Faced with a new pathogen, such as SARS-CoV-2, it is crucial to be able to forecast the case fatality rate (CFR) and the overall disease burden. Considerable effort has been invested towards developing a mathematical framework for predicting virulence evolution. Although many approaches accurately recapitulate complex outcomes, most rely on an assumed trade-off between CFR and infection rate. It is often impractical to empirically validate this constraint for human pathogens.

Methods: A compartment model with parameters tuning the degree to which symptomatic individuals are isolated and the duration of immunity is constructed and evaluated at both short timescales and at equilibrium.

Results: We reveal kinetic constraints whereby variation of multiple parameters in concert leads to decreased CFR and increased pathogen fitness, whereas independent variation of the parameters decreases pathogen fitness. Smallpox, SARS-CoV-2, and influenza are analyzed as diverse representatives of human respiratory viruses. We show that highly virulent viruses, such as smallpox, are often constrained by the host behavior, whereas moderately virulent viruses, such as SARS-CoV-2, appear to be typically constrained by the relationship between the duration of immunity and CFR.

Conclusions: Evolution of human respiratory epidemics appears to be often kinetically constrained and a reduction in CFR should not be assumed. These results agree with previous work demonstrating an increase in virulence for smallpox and further predict that SARS-CoV-2 is likely to continue presenting a substantial disease burden. Herd immunity against SARS-CoV-2 and viruses with similar life history traits might be unachievable without vaccination. However, partial isolation of symptomatic individuals can have a major effect on the epidemic dynamics not only by reducing the number of fatalities in the short term but also by changing the evolutionary trajectory of moderate CFR viruses towards reduced CFR.

\section{Open Peer Review \\ Approval Status \\ 1 \\ 2 \\ version 2 \\ (revision) \\ 29 Jul 2021 \\ version 1 \\ 04 Jun 2021

$\checkmark$
view \\ 1. Claus O. Wilke , The University of Texas, Austin, USA \\ 2. Kim Sneppen (iD), University of Copenhagen, Copenhagen, Denmark}

Any reports and responses or comments on the article can be found at the end of the article. 
Keywords

SARS-CoV2; influenza virus; smallpox virus; epidemic modeling; case

fatality ratio

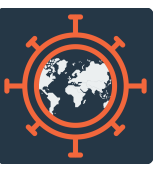

This article is included in the Emerging Diseases

and Outbreaks gateway.

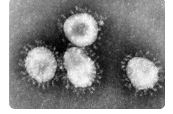

This article is included in the Coronavirus

collection.

Corresponding authors: Nash Rochman (nash.rochman@nih.gov), Eugene V Koonin (koonin@ncbi.nlm.nih.gov)

Author roles: Rochman N: Conceptualization, Formal Analysis, Investigation, Writing - Original Draft Preparation, Writing - Review \& Editing; Wolf YI: Conceptualization, Investigation, Writing - Review \& Editing; Koonin EV: Conceptualization, Investigation, Supervision, Writing - Review \& Editing

Competing interests: No competing interests were disclosed.

Grant information: U.S. Department of Health and Human Services. NDR, YIW, and EVK are supported by the Intramural Research Program of the National Institutes of Health (National Library of Medicine).

The funders had no role in study design, data collection and analysis, decision to publish, or preparation of the manuscript.

Copyright: ( 2021 Rochman $\mathrm{N}$ et al. This is an open access article distributed under the terms of the Creative Commons Attribution License, which permits unrestricted use, distribution, and reproduction in any medium, provided the original work is properly cited. The author(s) is/are employees of the US Government and therefore domestic copyright protection in USA does not apply to this work. The work may be protected under the copyright laws of other jurisdictions when used in those jurisdictions.

How to cite this article: Rochman N, Wolf YI and Koonin EV. Evolution of human respiratory virus epidemics [version 2; peer review: 2 approved] F1000Research 2021, 10:447 https://doi.org/10.12688/f1000research.53392.2

First published: 04 Jun 2021, 10:447 https://doi.org/10.12688/f1000research.53392.1 


\section{REVISED Amendments from Version 1}

In the new version of this manuscript, in response to the comments of the two reviewers, we have provided detailed additional explanations of the assumptions and limitations of the approach we employed to model the evolution of human respiratory virus epidemics. In particular, we included detailed explanations of the key concept of "kinetic constraints" on virus evolution that we introduce by analogy with activation anergy of chemical reactions and that reflects the inaccessibility of certain favourable states, such as those with low CFR, on the landscape of virus evolution. We also describe the limitations of our approach, such as the lack of invasion analysis, and present arguments for the general and flexible approach employed in this work. We additionally include an amended version of Table 1 explaining the variables and parameters of the model.

Any further responses from the reviewers can be found at the end of the article

\section{Background}

The case fatality rate (CFR) and infection rate largely determine the survival of both a pathogen and its host. The ideals CFR, for both the host and the pathogen, is zero. However, constraints imposed by the host behavior and pathogen biology prevent independent variation of the CFR and the infection rate, resulting in an evolutionary balancing act that can yield a wide range of host-pathogen relationships, from mutualism to host extinction. Predicting virulence evolution is of broad interest both from an evolutionary biology standpoint and for practical epidemiological purposes, and can only be accomplished through understanding the relevant constraints. Although comprehensive models of the evolution of virulence capable of describing complex environments have been developed, ${ }^{1-5}$ most studies to date impose constraints assumed from first principles and lacking experimental or empirical validation. ${ }^{6,7}$ Most commonly, a trade-off function is assumed ${ }^{8,9}$ between the infection rate and the CFR. Importantly, different definitions of virulence can lead to dramatically different predictions. ${ }^{10}$ In this work, we were primarily concerned with long-term trends and consider the CFR exclusively (not the rate of instantaneous mortality or other measures of virulence).

Individual hosts harboring high numbers of pathogen are more likely to die than those harboring lower numbers, but they also shed pathogen at increased rates and therefore could infect a greater number of new hosts. ${ }^{11}$ However, this straightforward picture is complicated by a landscape of opposite evolutionary outcomes. ${ }^{12}$ For example, vaccines that alleviate symptoms but still admit infection have been suggested to have induced selective pressures which favor the evolution of strains with increased virulence in malaria ${ }^{13,14}$ and decreased virulence for diphtheria and pertussis, ${ }^{15}$ likely due to differences in the cost of toxin production. ${ }^{16}$ This demonstrates how predictions of specific outcomes useful for informing public health intervention cannot be generalized across pathogens. The infectivity-virulence trade-off has been demonstrated for malaria. ${ }^{14}$ Although this could be true more generally, the existing data are scarce, and demonstrating this trade-off for most human pathogens is extremely difficult, due to the impracticality of comprehensive contact tracing. Many strategies to replace the explicit use of this trade-off have been discussed. ${ }^{11}$ One approach is to construct realistic models of within-host pathogen dynamics. ${ }^{17,18}$ This approach provides the ability to make nuanced predictions about specific pathogens and environmental conditions, but requires a deep understanding of the pathogen biology, which is often lacking, especially in the case of newly emergent pathogens.

Towards this goal, we explore a range of epidemiological outcomes for human pathogens modelled using available data on well-characterized respiratory viruses (see Table 1 for a description of keywords and model parameters). Our principal aim is to chart a "global phase space", indicating where endemic equilibrium is possible and to establish the bounds of potential evolutionary trajectories within this phase space without leaving that endemic equilibrium. Before proceeding, it is important to motivate the utility of studying endemic equilibrium at all. Endemicity within a host population is not a true equilibrium state and is subject to periodic outbreaks of variable severity. However, there is an important biological distinction between a pathogen that is capable of maintaining endemicity, and one that is not. Pathogens of the latter type could, in principle, still produce periodic outbreaks, resulting in cumulatively many infections; but in this case, the outbreaks must be predicated on zoonosis or extensive environmental transfer. Thus, once a pathogen achieves endemicity, we assume that most evolutionary trajectories resulting in a significant viral population must remain in a state of endemicity. We use the cumulative number of infections at endemic equilibrium, which represents the average behavior over periodic epidemics, as a proxy for the long term success of the pathogen.

It is further important to note that we do not explicitly conduct an invasion analysis. The ability of an emergent strain to invade depends on many factors that are not considered in the present model and the existence of a path within the global phase space does not guarantee that invasion along that path is probable for any particular virus. Conversely, if a path in the global phase space leads out of endemic equilibrium, this suggests that invasion along that path is most likely impossible. 
Table 1. Model parameters and key definitions.

\begin{tabular}{|c|c|}
\hline A & Probability (permanent) immunity is conferred after infection. \\
\hline B & Fraction of the symptomatic population which is well mixed (not isolated). \\
\hline $\mathrm{I} / \mathrm{i} / \mathrm{i}^{*}$ & \# of immune hosts / fraction / fraction at endemic equilibrium \\
\hline $\mathrm{S} / \mathrm{s} / \mathrm{s}^{*}$ & \# of susceptible hosts / fraction / fraction at endemic equilibrium \\
\hline $\mathrm{A} / \mathrm{a} / \mathrm{a*}^{*}$ & \# of asymptomatic and infectious hosts / fraction / fraction at endemic equilibrium \\
\hline $\mathrm{C} / \mathrm{c} / \mathrm{c}^{*}$ & \# of symptomatic and infectious hosts / fraction / fraction at endemic equilibrium \\
\hline$k_{B}$ & Birthrate \\
\hline$k_{D}$ & Baseline deathrate unrelated to infection. \\
\hline $\mathrm{k}_{\text {contact }}$ & Rate of host-host contact \\
\hline$P($ infect $)$ & Probability of infection given contact between a susceptible and an infected host \\
\hline $\mathrm{k}_{\mathrm{I}}$ & $\mathrm{k}_{\text {contact }} \mathrm{P}$ (infect), the infection rate. \\
\hline$k_{p}$ & Rate of progression from asymptomatic to symptomatic. \\
\hline $\mathrm{k}_{\mathrm{R}}$ & Rate of recovery from infection. \\
\hline$k_{D V}$ & Rate of death due to infection. \\
\hline $\mathrm{k}_{\mathrm{L}}$ & $(1-\alpha) \mathrm{k}_{R}(A+C) / \mathrm{I}$ (the rate at which immunity is lost, may be used at endemic equilibrium) \\
\hline * & Notation indicates reference to endemic equilibrium. \\
\hline $\begin{array}{l}\text { Disease Free } \\
\text { Equilibrium }\end{array}$ & No pathogen remains in the host population (pathogen extinction) \\
\hline Endemic Equilibrium & A stable fraction of the host population remains infected at large times. \\
\hline Host Population Crisis & Less than $10 \%$ of the initial host population remains. \\
\hline Susceptible Bottleneck & Less than $10 \%$ of the population is susceptible to infection. \\
\hline Unsustainable & The host population size decreases at endemic equilibrium. \\
\hline CFR & Case fatality rate. \\
\hline Fitness & In the context of this work, the total number of infected hosts. \\
\hline Kinetically Constrained & $\begin{array}{l}\text { A regime exists in the parameter space where the total number of infected hosts is } \\
\text { larger and the CFR is lower than in the observed regime; however, any evolutionary } \\
\text { trajectory that is available to reach that superior domain of the parameter space } \\
\text { requires the perturbation of multiple parameters. }\end{array}$ \\
\hline $\begin{array}{l}\text { Thermodynamically } \\
\text { Constrained }\end{array}$ & $\begin{array}{l}\text { No parameter regime exists where the total number of infected hosts is larger and } \\
\text { the CFR is lower than in the observed regime. }\end{array}$ \\
\hline
\end{tabular}

With these limitations acknowledged, surveying the life history characteristics of common human respiratory viruses (Table 2) 20-28 suggests the existence of nontrivial bounds within the global phase space. In particular, there exists an inverse relationship between the time during which the host is asymptomatic but infectious and the CFR. ${ }^{19-27}$ Furthermore, historically, vaccination against lower-CFR viruses, such as influenza, relative to higher-CFR viruses, such as smallpox, has posed a greater challenge. Although this is in part due to a comparatively low economic incentive, the positive correlation between CFR and the duration of immunity is likely to be another important contributor. Through this approach, we are able to identify broad evolutionary constraints on pathogen evolution.

In addition to being difficult to validate through direct experiments or other empirical evidence, the transmission/ virulence trade-off or related trade-offs are often analyzed under an implicit assumption that virulence evolution is a onedimensional problem: pathogens can be more transmissible and more virulent or less transmissible and less virulent. Virulence and transmissibility are only two of many co-dependent life-history characteristics of a pathogen, and although a single trade-off function might often be a reasonable model over short evolutionary distances, it appears highly likely that, on longer time scales, no single constraint would hold globally. In other words, in principle, any combination of life history traits that admits endemic equilibrium is biologically feasible, and the critical question then becomes whether there is an accessible evolutionary path connecting any two such points in the life-history phase space. Acknowledging the limitations of this approach as discussed above, we identify global barriers limiting evolutionary trajectories which would otherwise lead to the reduction in CFR for human respiratory viruses. 
Table 2. Human respiratory viruses with substantial health impact.

\begin{tabular}{|c|c|c|c|c|}
\hline Name & $\begin{array}{l}\text { Case } \\
\text { Fatality } \\
\text { Rate }\end{array}$ & $\begin{array}{l}\text { Time Asym. N. } \\
\text { Inf. (days) }\end{array}$ & $\begin{array}{l}\text { Asym. } \\
\text { Inf. }\end{array}$ & Time Sym. (days) \\
\hline MERS-COV & 0.35 & $2-14$ & minimal & up to 90 \\
\hline $\begin{array}{l}\text { Variola major } \\
\text { (smallpox) }\end{array}$ & 0.3 & $7-19$ & $\begin{array}{l}\text { assumed } \\
\text { no }\end{array}$ & $9-11$ \\
\hline SARS-CoV & 0.15 & 6 & $\begin{array}{l}\text { assumed } \\
\text { no }\end{array}$ & 20 \\
\hline $\begin{array}{l}\text { Measles } \\
\text { morbilivirus }\end{array}$ & $0.04-0.1$ & $6-21$ & $\begin{array}{l}<\text { one } \\
\text { week }\end{array}$ & $6-8$ \\
\hline SARS-CoV-2 & $<0.02$ & $5-14$ & $\begin{array}{l}<\text { one } \\
\text { week }\end{array}$ & 21 \\
\hline Influenza & $<0.002$ & 2 & $\begin{array}{l}<\text { one } \\
\text { week }\end{array}$ & 3 \\
\hline Mumps rubulavirus & near 0 & $16-18$ & $\begin{array}{l}<\text { one } \\
\text { week }\end{array}$ & $6-9$ \\
\hline Rubella virus & near 0 & $14-18$ & $\begin{array}{l}\text { up to two } \\
\text { weeks }\end{array}$ & $\begin{array}{l}\text { largely asymptomatic, symptomatic } \\
\text { period largely not infectious }\end{array}$ \\
\hline Rhinovirus & near 0 & \multicolumn{3}{|c|}{$\begin{array}{l}\text { Time course varies, can be infectious for weeks, or wholly asymptomatic, } \\
\text { or symptomatic on the first day of infectious period. }\end{array}$} \\
\hline $\begin{array}{l}\text { Human respiratory } \\
\text { syncytial virus }\end{array}$ & near 0 & \multicolumn{3}{|c|}{ Can be wholly asymptomatic } \\
\hline $\begin{array}{l}\text { Human } \\
\text { parainfluenza }\end{array}$ & near 0 & \multicolumn{3}{|c|}{ Can be wholly asymptomatic } \\
\hline Alphacoronavirus & near 0 & \multicolumn{3}{|c|}{ Time course varies, can be wholly asymptomatic } \\
\hline
\end{tabular}

We describe these barriers to the evolution of CFR as "kinetic constraints", which is analogous to the activation energy for an energetically favorable chemical reaction.

Consider a simple, reversible chemical reaction between reactant, $\mathrm{R}$, and product, $\mathrm{P}$. When sufficient energy, A1, is applied, $\mathrm{R}$ is converted to $\mathrm{P}$. This is the activation energy of the forward reaction. Now consider that $\mathrm{P}$ is much more stable than $\mathrm{R}$ so that $\mathrm{P}$ cannot be converted back to $\mathrm{R}$ unless $10 \times \mathrm{A} 1$ energy is applied. This is the activation energy of the reverse reaction. Given sufficient energy and time, the system will reach an equilibrium in which the quantity of $\mathrm{P}$ is 10 -fold higher than the quantity of R. However, if insufficient energy is applied, less than A1, the system will remain almost wholly composed of $\mathrm{R}$. Under these conditions, the reaction is kinetically constrained. Now suppose that $\mathrm{P}$ is much less stable than $\mathrm{R}$ so that $\mathrm{P}$ is converted back to $\mathrm{R}$ whenever $0.1 \times \mathrm{A} 1$ energy is applied. In this case, even given sufficient energy and time, the system will remain in a state in which the amount of $\mathrm{R}$ is at least 10-fold greater than the amount of P. Under these conditions, the (forward) reaction is thermodynamically constrained.

The evolution of pathogen life history traits can be described as a system of reversible reactions where the "stability" of each set of life history traits is estimated by the total number of infected hosts at endemic equilibrium. The evolution of decreased CFR would be thermodynamically constrained if it were impossible to simultaneously increase the total number of infected hosts and decrease CFR. However, because the maximum size of the viral population and the minimum cost to the host is achieved at a CFR of 0 (see below), evolution of CFR is never thermodynamically constrained. In contrast, evolution of CFR would be kinetically constrained if there existed a combination of lifehistory characteristics, for which the total number of infected hosts was higher and CFR was lower, but traversing the evolutionary paths to that state would require simultaneously modifying multiple parameters, some of which are determined by the host behavior, constituting a high "activation energy".

Additionally, the evolution of decreased CFR could be kinetically constrained by many barriers to invasion, which might vary among pathogens or among host populations for the same pathogen. Even without the consideration of specific barriers to invasion, we are able to demonstrate global kinetic constraints to the evolution of decreasing CFR for human respiratory viruses. We show that, for high-CFR viruses such as smallpox, the relationship between the infection rate and CFR is likely a kinetic constraint, rather than an actual trade-off, whereas for intermediate-CFR viruses, such as 
SARS-CoV-2, there is a kinetic constraint between immunity and CFR. Analysis of such constraints could open avenues for prediction of epidemic outcomes and quantitative validation of such predictions.

\section{Methods}

The introduction of a novel pathogen into a host population is likely to result in one of three outcomes, whenever the number of infections is not too small and the basic reproduction number is greater than one, $R_{0}>1$ (otherwise disease-free equilibrium is achieved). 1) The number of fatalities is large enough for the pathogen to wipe out the host population (as a result, the pathogen itself also goes extinct) (Figure 1A). 2) The number of infections is large enough, and the number of fatalities is small enough, such that the pathogen creates a bottleneck in the susceptible host population. With all hosts either infected or immune, the pathogen is eliminated from the host population (Figure 1B). 3) The number of infections is small enough such that a bottleneck in the susceptible population is avoided and long-term persistence within the host population is possible (Figure 1C). If the basic reproduction number is less than one, disease-free equilibrium is achieved. These three outcomes can occur on vastly different timescales, with a host wipeout or susceptible bottleneck occurring much faster than the rate at which equilibrium is approached. Throughout this work, we simulate each epidemic until one of these three outcomes is reached. With $R_{0}>1$, a state of stable endemic equilibrium can be reached where the fraction of the host population susceptible to infection remains constant (up to fluctuations). However, if the pathogen-associated fatality exceeds the birth-rate of the host population, the host-pathogen relationship becomes unsustainable in the long term. Such an unsustainable relationship would pose an intense selective pressure on the host population, likely resulting in the extinction of the pathogen through the modification of host behavior or the emergence of resistant hosts.

Which of these courses an epidemic follows, largely depends on the balance of four factors. 1) The frequency of host-host interaction, with or without isolation of infected individuals or prophylactic quarantine (Figure 1D). 2) The likelihood of an uninfected host to become infected after interacting with an infected host (Figure 1E). 3) The CFR of the pathogen, that is, the likelihood that an infected host will die as a result of infection (Figure 1F). 4) The duration of host immunity post infection (Figure 1G). Although the effect of tuning each of these parameters often appears obvious, for example, decreasing the frequency of host-host interaction almost always decreases the number of infections - many counterintuitive observations become apparent. For example, decreasing the frequency of host-host interaction under conditions that would otherwise lead to a bottleneck in the susceptible population (Figure 1B) can result in long term persistence within the host population which, avoiding pathogen extinction, ultimately increases the total number of infections
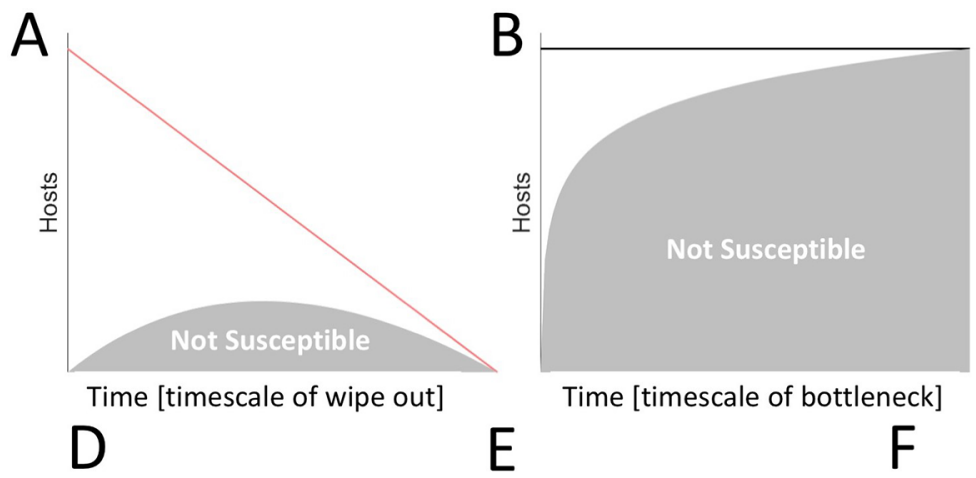

Time [timescale of bottleneck]
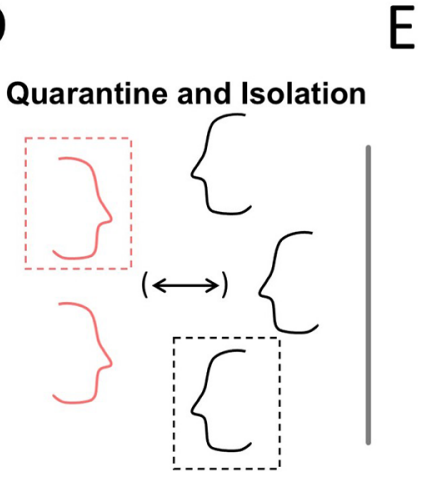
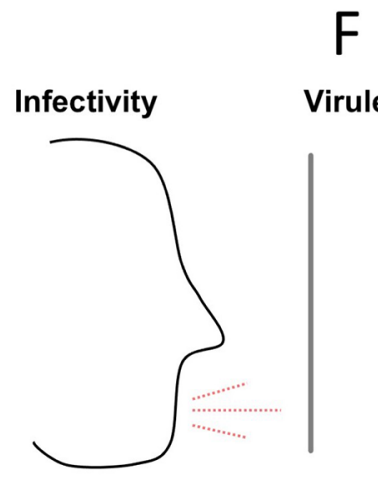

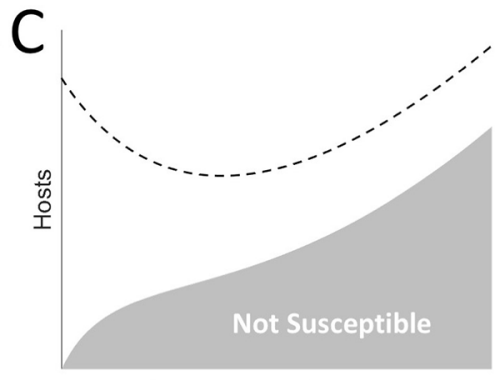

Time [timescale of equilibrium]
Virulence and Mortality Immunity
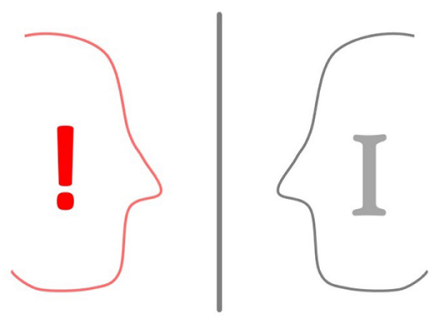

Figure 1. Key factors that determine epidemiological outcomes. A-C. Cartoons representing the three potential outcomes for an epidemic beyond trivial disease-free equilibrium. Lines indicate total number of hosts, shaded areas indicate the fraction of hosts which are not susceptible. A. Host extinction. B. A bottleneck in the susceptible population. C. A balance between infections, CFR, and birthrate. D. Host interaction, quarantine, and isolation. E. Probability of Infection Following Host Contact (Infectivity). F. CFR. G. Immunity. 
sustained over time. In an effort to better delineate how the host-pathogen relationship varies across the space of these factors, we constructed the following model.

Hosts are assigned one of the four possible states: 1) immune, $I ; 2$ ) susceptible, $S ; 3$ ) asymptomatic, $A$; and 4 ) symptomatic or "clinical", $C$. New hosts are assumed to be born susceptible at a rate, $k_{B}$, and a baseline death rate, $k_{D}$, is assumed constant across all compartments. Susceptible hosts can be infected by coming into contact with either asymptomatic or clinical hosts. Asymptomatic hosts either recover at rate, $k_{R}$, or progress to the clinical compartment at rate, $k_{P}$. Clinical hosts either recover at rate, $k_{R}$, or die due to the pathogen at rate, $k_{D V}$.

In the model, as written, recovery confers permanent immunity with probability, $\alpha$. This is done for mathematical convenience. Under many if not most circumstances, it is more realistic to assume recovery confers immunity which is gradually lost at a constant rate, $k_{L}$. At endemic equilibrium, one may solve for the parameter $k_{L}=(1-\alpha) k_{R}(A+C) / I$ (see Appendix $\mathrm{A}$ in the extended data ${ }^{28}$ ) as a function of $\alpha$; however, the dynamics approaching equilibrium are different for the two formalisms. Thus, when analyzing short-term dynamics, we assume that only a negligible portion of the population has lost immunity and fix $\alpha=1$.

The population is assumed to be well mixed, with the exception of the clinical compartment, a fraction $(1-\beta)$ of which is isolated and cannot infect susceptible hosts, which is equivalent to modelling a decreased contact rate for the entire clinical compartment. Furthermore, $\beta<1$ indicates a state of isolation only for symptomatic individuals. This is not equivalent to the prophylactic quarantine of asymptomatic individuals and does not imply the imposition of emergency public health measures.

The rate at which susceptible hosts become infected depends on both the host behavior and the pathogen biology. This compound rate is the triple product of the rate of contact between hosts, the fraction of the population (not isolated) which is infected, and the probability of infection upon contact: $k_{\text {contact }} \frac{A+\beta C}{I+S+A+\beta C} P($ infect $), k_{I} \equiv k_{\text {contact }} P($ infect $)$, and may freely vary; however, change of either of these parameters is equivalent within the model. For simplicity, we only vary the product, throughout this work, and without loss of generality, fix to describe variation in as variation in alone. has units of 1/time and increasing is equivalent to decreasing the mean time between host-host contacts, as displayed in the figures. Lower values of $P($ infect $)$ would not change the bounds on the global phase space or the qualitative trends displayed in any figures; however, they would correspond to higher values of host contact rates than those that appear on the figure axes. This yields the system of ordinary differential equations (Figure 2A):

$$
\left[\begin{array}{l}
I \\
S \\
A \\
C
\end{array}\right]^{\prime}=\left[\begin{array}{cccc}
-k_{D} & 0 & \alpha k_{R} & \alpha k_{R} \\
k_{B} & k_{B}-k_{D}-k_{I} \frac{A+\beta C}{I+S+A+\beta C} & k_{B}+(1-\alpha) k_{R} & k_{B}+(1-\alpha) k_{R} \\
0 & k_{I} \frac{A+\beta C}{I+S+A+\beta C} & -\left(k_{R}+k_{D}+k_{P}\right) & 0 \\
0 & 0 & k_{P} & -\left(k_{R}+k_{D}+k_{D V}\right)
\end{array}\right]\left[\begin{array}{l}
I \\
S \\
A \\
C
\end{array}\right]
$$

With two infected states (asymptomatic and clinical) and isolation, ISAC is a simple model within the range of models $^{29-31}$ that have been developed in response to the SARS epidemic.

Short-term dynamics can be inferred from this system of equations, to establish whether a bottleneck in the size of the susceptible population (Figure 1B), or the entire population representing a host crisis (Figure 1A), occurs. For the analysis of dynamics within this period, we assume that only a negligible portion of the host population has gained and subsequently lost immunity, setting $\alpha=1$. To study this case, we explicitly simulate an epidemic beginning with an initial condition where the size of the infected compartments is five orders of magnitude lower than the value corresponding to the endemic equilibrium, in order to capture realistic dynamics for the emergence of a new pathogen. The basic reproduction number, $R_{0}$, for this model can be derived through the construction of next generation matrices ${ }^{32}$ (see Appendix B in the extended data):

$$
R_{0}=\frac{k_{1}}{k_{R}+k_{D}+k_{P}}\left(1+\frac{\beta k_{P}}{k_{R}+k_{D}+k_{D V}}\right)
$$

Short-term dynamics are sometimes determined simply by the $R_{0}$, value and when $R_{0}<1$, the pathogen will go extinct. When $R_{0}>1$, a wide range of dynamics are possible including short term bottlenecks and long-term endemic equilibrium. 


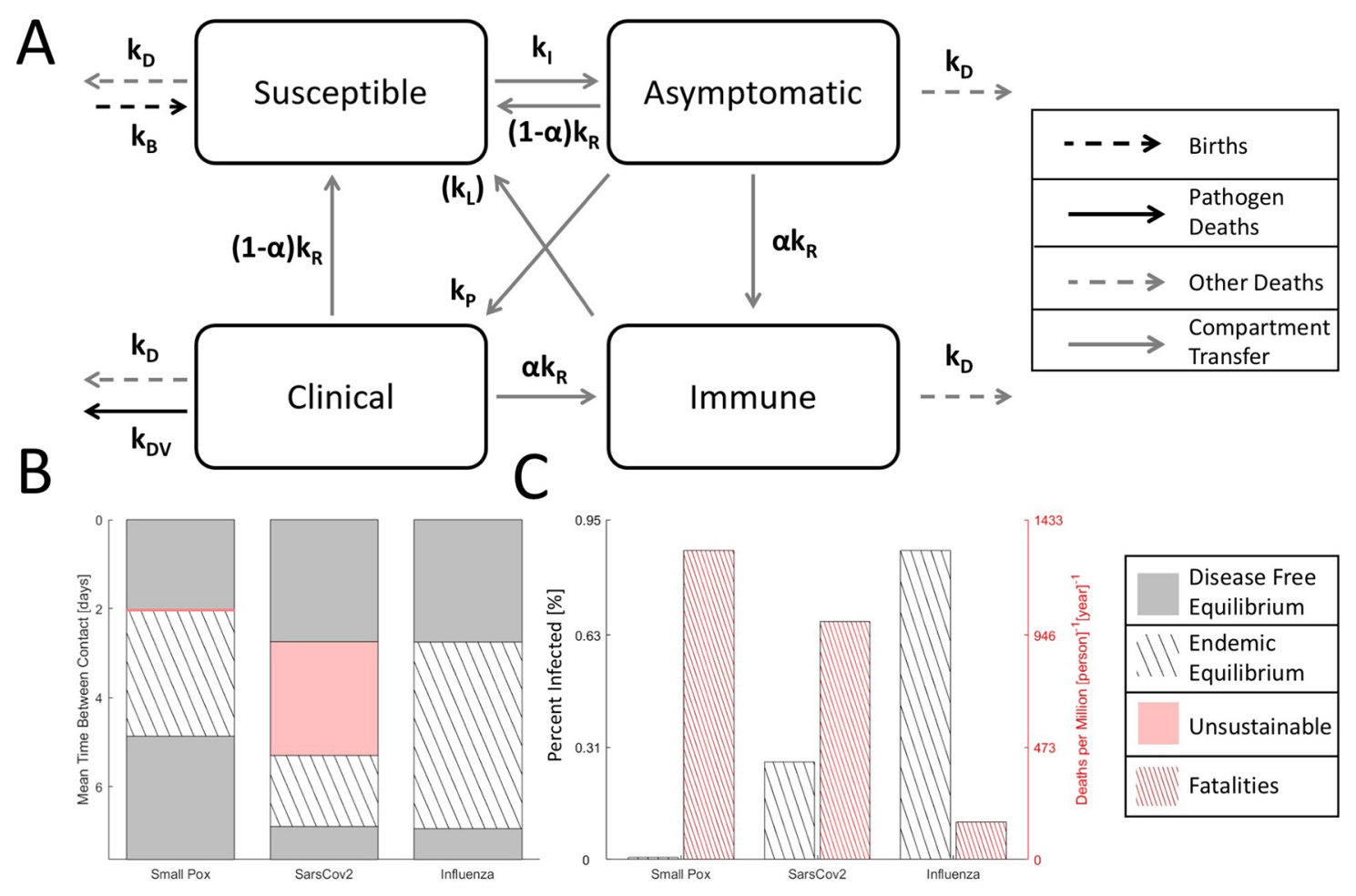

Figure 2. Four compartment model of an epidemic. A. Cartoon of the compartment model. Note that, in the model, permanent immunity is gained with probability $\alpha$ for mathematical convenience. Alternatively, temporary immunity can be gained with a probability of 1 and lost at rate $k_{L}$. Although the dynamics are different, the corresponding endemic equilibria can be identified by expressing $k_{L}$ as function of $\alpha$ and compartment frequencies. B. Disease free equilibrium (gray), endemic equilibrium (striped), and unsustainable (red) regions for three analyzed viruses over a range of host contact rates. The unsustainable region for smallpox-like viruses is narrow and not shown to scale. C. For endemic equilibrium (contact rates correspond to the midrange shown in B), the fraction of the host population infected and the death rate. Note that, representing equilibrium, the choice of timescale (years) is arbitrary.

In many cases when $R_{0}>1$ and a bottleneck is avoided, we are interested in examining the stationary points for the above system of equations. Unique, stable stationary points correspond to endemic equilibrium. While uniqueness and stability have been proven for a very broad class of compartment models, ${ }^{33,34}$ more generally, this can be numerically demonstrated for any stationary point as is done throughout this work. At endemic equilibrium, the fraction of the total population, $N$, in each compartment, $X$, is constant: $\left(\frac{X}{N}\right)^{\prime}=x^{\prime}-x n^{\prime}=0$ (we denote the fraction of each compartment with lowercase letters). Endemic equilibrium corresponds to a stable stationary point for this system of equations. We represent values corresponding to endemic equilibrium with a "*", to reflect this. We do not assume a constant size of the total population; however, the results of this simplifying assumption are presented in Appendix C (extended data). More generally, $n^{\prime}=k_{B}-k_{D}-c k_{D V}$ which yields:

$$
0=\left[\begin{array}{c}
I / N \\
S / N \\
A / N \\
C / N
\end{array}\right]^{\prime}=\left[\begin{array}{cccc}
c^{*} k_{D V}-k_{B} & 0 & \alpha k_{R} & \alpha k_{R} \\
k_{B} & c^{*} k_{D V}-k_{I} \frac{a^{*}+\beta c^{*}}{i^{*}+s^{*}+a^{*}+\beta c^{*}} & k_{B}+(1-\alpha) k_{R} & k_{B}+(1-\alpha) k_{R} \\
0 & k_{I} \frac{a^{*}+\beta c^{*}}{i^{*}+s^{*}+a^{*}+\beta c^{*}} & c^{*} k_{D V}-\left(k_{B}+k_{R}+k_{P}\right) & 0 \\
0 & 0 & k_{P} & c^{*} k_{D V}-\left(k_{B}+k_{R}+k_{D V}\right)
\end{array}\right]\left[\begin{array}{c}
i^{*} \\
s^{*} \\
a^{*} \\
c^{*}
\end{array}\right]
$$

We are primarily interested in establishing the fraction of hosts that are infected $\left(i^{*}+c^{*}\right)$ and the number of deaths caused by the pathogen per year $\left(k_{D V} c^{*}\right)$ at endemic equilibrium and, generally, aim to algebraically solve this system of equations for the relative size of each compartment. This system can be solved to yield a fourth order polynomial with respect to $c^{*}$ (we used the MATLAB symbolic toolbox (RRID:SCR_001622), ${ }^{35}$ an open-source alternative is Julia, see Appendix D in extended data); however, it is cumbersome enough that a numerical solution appears preferable and establishing opportunities for linearization is desirable. 
Endemic equilibrium requires a constant or growing population, limiting the total number of deaths due to infection that can be sustained by the host population per unit time and, accordingly, provides an upper bound for the size of the clinical compartment. $\frac{k_{B}-k_{D}}{k_{D V}} \geq c^{*} ; \frac{k_{B}-k_{D}}{k_{R}} \geq c \frac{k_{D V}}{k_{R}}$. For human populations and pathogens, it is reasonable to assume that the birth rate is much lower than the recovery rate, $\frac{k_{B}, k_{D}}{k_{R}} \ll 1$, yielding the limit: $c^{*} \frac{k_{D V}}{k_{R}} \ll 1$. Conversely, populations with higher birth rates admit endemic equilibrium for more virulent pathogens, and even among human populations, the host population size plays a critical role in the determination of whether acute pathogens persist. ${ }^{18}$

Thus, for an endemic equilibrium to exist, either the clinical compartment has to be very small or the death rate due to the virus has to be very low compared to the recovery rate. This allows us to linearize the model with respect to either the size of the clinical compartment, for pathogens with high CFR, or the ratio of the death rate to the recovery rate, for low CFR pathogens. For the high CFR case, we solve the above system of algebraic equations to first order in $c^{*}$ (e.g. $\left(c^{*}\right)^{2}+c^{*} \sim c^{*}$, where by definition $0<c^{*}<1$, see Appendix $\mathrm{E}$ in the extended data). This linearized model admits an analytic solution for endemic equilibrium, given a sufficiently large $\alpha$ corresponding to at least partial immunity whenever $R_{0}>1$. In this case, the additional constraint $k_{P}>k_{R}+k_{D V}$, which largely holds for the pathogens considered here, is applied for convenience. For the low CFR case, we solve the above system of equations to first order in $\frac{k_{D V}}{k_{R}}$ (see Appendix F in the extended data). Here the stability of the solution depends on the parameters (it does not belong to the class of models referenced above). Both the stability and the general solution for the critical point are calculated numerically (see Appendix G in the extended data); however, analytic forms for endemic equilibria in the stricter limit $c^{*} \frac{k_{D V}}{k_{B}} \ll 1$ are provided as well.

Parameters are referenced against those selected to represent three respiratory pathogenic viruses: smallpox, SARSCoV-2, and influenza representing a range of phenotypes (Table 2), ${ }^{19-27}$ in agreement with the ranges reported for each parameter in the selected references. Note that the fifth column of Table 2 represents the total duration of symptoms, which can be substantially longer than the duration of the symptomatic and infectious period. The latter but not the former is a model parameter. We emphasize, however, that given the generality of the model, we are unable to make specific predictions for any of these viruses in a defined host population. We instead contrast the results for "smallpox-like" (high CFR), "SARS-CoV-2-like" (moderate CFR), and "influenza-like" (low CFR) viruses. Here, $k_{B}$ and $k_{D}$ are fixed whereas $k_{I}$ is varied; however, we will emphasize that throughout the text, we consider $P($ infect $)=1$ and describe variation in $k_{I}$ as variation in $k_{\text {contact }} . k_{R}$ and $k_{P}$ are fit to an estimated disease course for a host which is asymptomatic and infectious for the time $t_{P}=1 / k_{P}$, and symptomatic and infectious for the time $t_{R}=1 / k_{R}$ before recovering. For simplicity, death due to infection is assumed to occur only during the symptomatic and infectious phase: $k_{D V}=\frac{C F R}{1-C F R} k_{R}$. Smallpox is modelled with a CFR of 30\%, mean time to recovery 1 week, and no asymptomatic and infectious period. SARS-CoV-2 is modelled at a CFR of $1 \%, 1$ week recovery period, and 3 days asymptomatic and infectious. Influenza is modelled at a CFR of $0.05 \%, 1$ week recovery period, and 3 days asymptomatic and infectious. Smallpox infection is assumed to confer permanent immunity. SARS-CoV-2 and influenza infections are assumed to confer immunity for one year on average. A constant birthrate of 2.5 births per 2 people over 100 years and death rate of one death per person over 100 years is assumed. It should be emphasized that these model parameters are selected to broadly represent these three disparate index viruses and that our specific parameter choices are not necessarily an accurate reflection of any specific outbreak. In particular forSARS-CoV-2, given its recent identification, the most realistic parameter regime is subject to change.

\section{Results}

Endemic equilibrium, where an unchanging fraction of the host population remains infected in the long term, is bounded within a range of (host) contact rates (Figure 2B). When the mean time between contacts is too long and $k_{I}$ is too low, $k_{I}=\left(k_{R}+k_{D}+k_{P}\right)\left(1+\frac{\beta k_{P}}{\left(k_{R}+k_{D}+k_{D V}\right)}\right)^{-1}, R_{0}<1$, the pathogen goes extinct, and disease-free equilibrium, where there is no remaining pathogen, is reached. Likewise, when contacts are too frequent, a bottleneck in the susceptible population occurs (Figure 1B, here assumed to drop down to $10 \%$ of the total population), and the pathogen goes extinct. For some viruses, such as smallpox-like and SARS-CoV-2-like viruses, but not influenza-like viruses, a range of contact rates exists where the fraction of the infected population at endemic equilibrium is large enough so that the death rate exceeds the birth rate and the host-virus relationship is unsustainable in the long term, resulting in population decline without decreased pathogen CFR or modified host behavior. This range is very narrow for smallpox-like viruses but notably broad for SARS-CoV-2-like viruses (Figure 2B, not shown to scale) encompassing a wider range of host behavior than endemic equilibrium. The existence of this region in the parameter space implies that herd immunity, without vaccination, might be impossible to reach. In the middle range of contact rates admitting endemic equilibrium (Figure 1C), the decreased fraction of the infected population for all three examined viruses is offset by increasing CFR leading to an increased death rate. Under these model assumptions, the yearly death rate for a SARS-CoV-2-like virus is approximately 6-times that of an influenza-like virus. 
To examine an expanded two-dimensional phase space, we allowed the CFR to vary from $10 \%$ to $100 \%$ for smallpoxlike viruses, with no asymptomatic spread and permanent immunity, and from $0 \%$ to $10 \%$, for SARS-CoV-2-like and influenza-like viruses, with asymptomatic spread and temporary immunity (Figure 3). In Figure 3, the solid line separating the smallpox-like regime and the SARS-CoV-2-like regime denotes this difference in the duration of immunity. The dashed line between the influenza-like regime and the SARS-CoV-2-like regime indicates that there is no difference in the duration of immunity. The CFR, host-host contact rate, and the duration of immunity, which is explored in the next figure, are the three most relevant parameters for the study of human pathogenic respiratory viruses as becomes apparent from the observed variability among known pathogens (Table 2). However, it is important to recognize the effects of the rate of recovery on the global phase space as well. Throughout this work, we assume a 1-week recovery period. In a supplemental figure (see extended data), we display the results for two additional values ( 3 days/2 weeks, Figure S1. A/B). The dependency on CFR is remarkably similar across all three recovery regimes, but as one would expect, slower rates of recovery correspond to lower host-host contact rates required to achieve endemic equilibrium.

Note that disease-free equilibrium near the top of Figure 3 indicates a susceptible bottleneck (as illustrated in Figure 1B). As the CFR increases, both threshold contact rates, corresponding to $R_{0}=1$ and to the bottleneck in the susceptible population respectively, increase and the range admitting endemic equilibrium narrows. Across much of the phase space, the host-pathogen relationship is unsustainable, and at very high CFR, the total host population falls below $10 \%$ (dark red) in the initial phase of the epidemic, signaling possible extinction at short timescales. The contours within the region corresponding to the endemic equilibrium indicate the total size of the infected host population (which is proportional to the size of the viral population and constitutes our measure of virus fitness). Within this region, increasing contact rate and decreasing CFR increases the size of the infected population. At extremely high CFR, the gradient points primarily in the direction of decreasing CFR, and at low contact rates, the gradient points primarily in the direction of increasing contact rate.

Throughout most of the region in this phase space corresponding to endemic equilibrium for smallpox-like viruses (Figure 3), moving down and to the left increases the size of the infected population and suggests evolution towards decreased CFR. However, this is not the case at the top-right corner representing viruses with extremely high CFR and extremely high contact rates (or alternatively viruses with a much higher likelihood of infection following host contact). Such, hypothetical, viruses would have an unsustainable relationship with the host population if CFR decreased (and

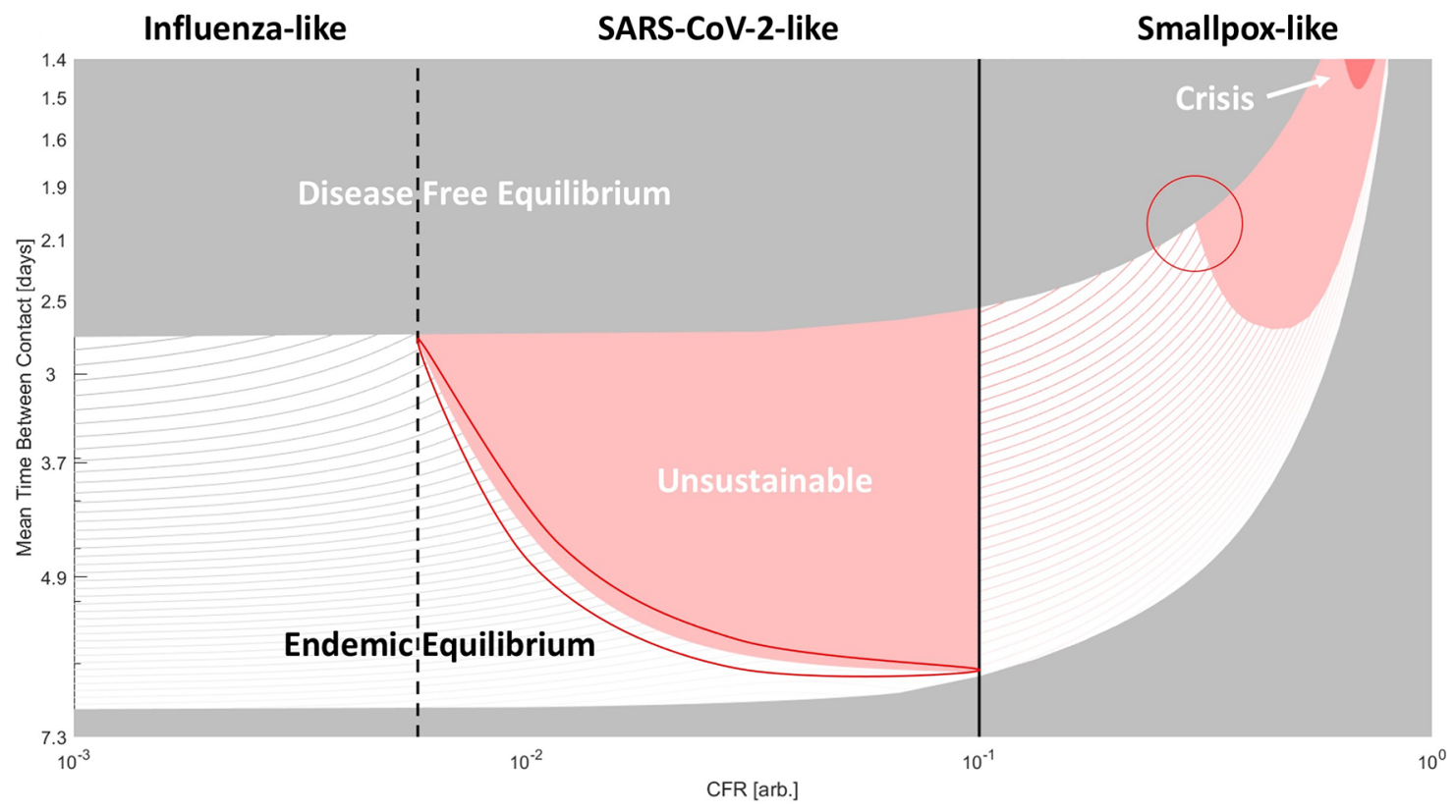

Figure 3. Phase diagram of epidemiological outcomes. The CFR and the contact rate were varied. For variable recovery rate, see Figure S1. Disease-free equilibrium (gray), endemic equilibrium (striped), unsustainable (red), and host population crisis (loss of $90 \%$, dark red) regions are shown. For CFR $>10 \%$, smallpox-like features are assumed. For CFR $<10 \%$ SARS-CoV-2/influenza-like features are assumed (these viruses are only distinguished within the model by CFR). Lines within the region bounding endemic equilibrium indicate contours for the percentage of the population infected $\left(a^{*}+c^{*}\right)^{*} 100$. Darker color corresponds to higher values. Two regions subject to kinetic constraints are outlined. 
infected hosts were less likely to die before interacting with uninfected hosts) and are, in a sense, kinetically constrained, likely, by the host behavior. The population size of such viruses would dramatically increase if the CFR was reduced but would remain in endemic equilibrium only if contact rates or the rate of infection simultaneously decreased. Smallpox evolution could be similarly kinetically constrained. On the phase diagram for high-CFR viruses, smallpox, with a CFR of $30 \%$, is located near the triple point for host populations with high contact rates where the regions of disease-free equilibrium, endemic equilibrium and unsustainability meet (highlighted in Figure 3). To increase the size of the infected population, both CFR and contact rate must decrease, whereas decreasing only the CFR ultimately results in disease-free equilibrium when such a virus enters new host communities, due to a bottleneck in the size of the susceptible population.

The corresponding triple point located at the boundary between influenza-like and SARS-CoV-2-like viruses lacks this property. For these viruses, decreasing CFR increases the size of the infected population throughout the parameter space. However, the boundary highlighted within the SARS-CoV-2-like diagram (Figure 3) represents a different kinetic constraint, this one, between immunity and CFR. While decreasing CFR below $10 \%$ minimally affects the threshold contact rates $\left(R_{0}=1\right.$ and susceptibility bottleneck), varying the duration of immunity has a dramatic impact on the phase diagram (Figure 4A). As the duration of immunity decreases, with $\alpha=0.01$ corresponding to approximately 1.5 years at the phase boundary, the endemic equilibrium region shrinks substantially.

As is apparent from the analytic solutions given in the extended data and illustrated for a hypothetical virus with a CFR of zero (other parameters matching influenza and Sars-Cov-2) and the maximum admitted contact rate (Figure 4B), decreasing the duration of immunity dramatically increases the size of the infected population at endemic equilibrium across the parameter regimes. While typically viewed from a host-centric perspective, immunity is almost always necessary for the maintenance of endemic equilibrium and thus required for maintaining large viral populations over long time scales. Consider a SARS-CoV-2-like virus near the highlighted boundary in Figure 3. Suppose this virus acquires an adaptation enabling immune evasion and thus decreasing the mean duration of immunity post infection. The size of the infected population will increase and, being near the boundary, the host-pathogen relationship will become unsustainable. This is another example of a kinetic constraint. Decreasing both CFR and the duration of immunity increases the size of the infected host population. However, maintaining a stable host-pathogen relationship and large numbers of infected hosts in the long term requires that the reduction in CFR is proportional to the reduction in immune duration. Otherwise, the overall death rate for the host population might increase despite a reduction in pathogen CFR. Notably, decreasing the

A

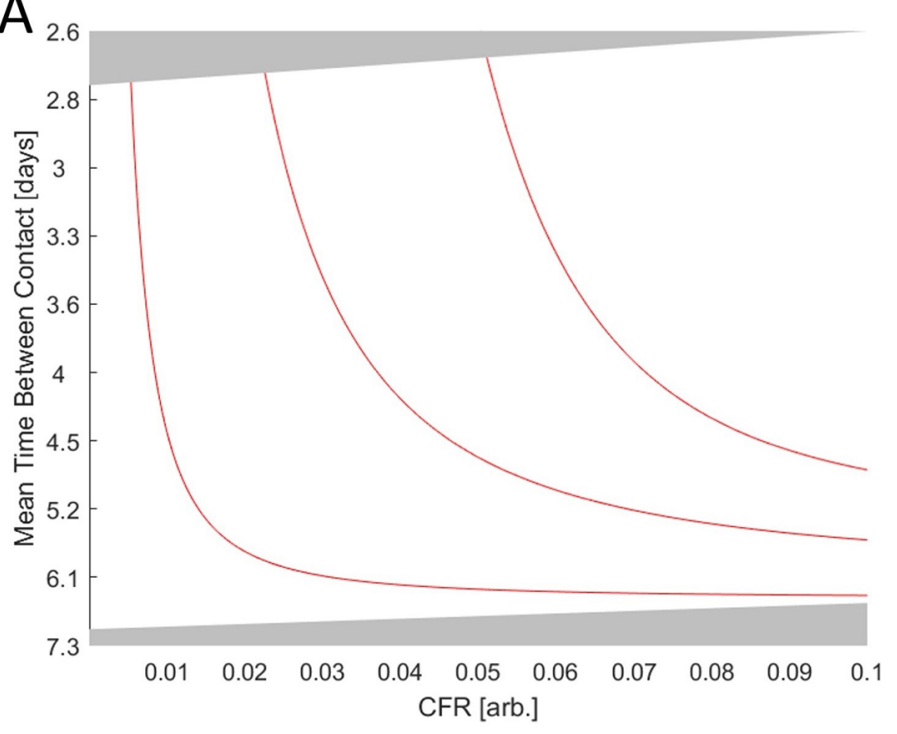

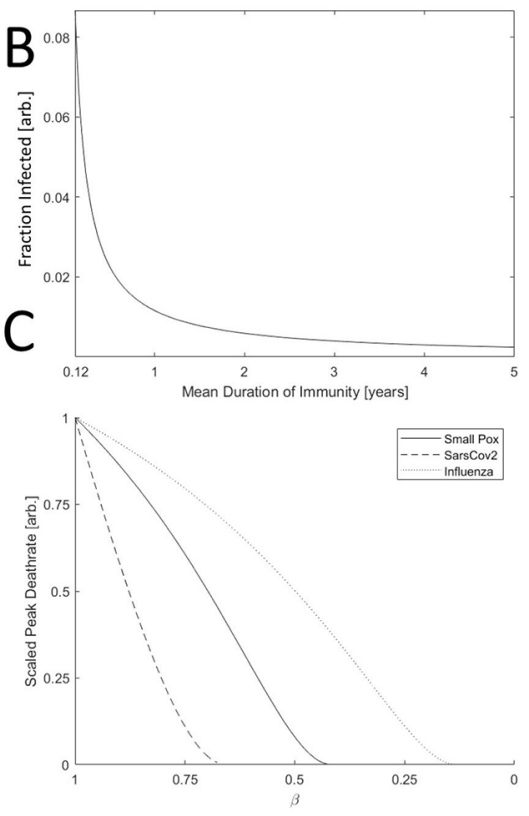

Figure 4. The role of immunity in epidemic evolution. A. Phase diagram of a SARS-CoV-2-like virus varying CFR and contact rate. Red lines indicate the boundary between endemic equilibrium and unsustainable for $\alpha \in$ $\{0.01,0.045,0.08\}$ regions corresponding to approximately $\{1.5,7,13\}$ years of immunity, respectively. $B$. The fraction of the population infected, $\left(a^{*}+c^{*}\right)$, vs the duration of immunity for a hypothetical virus with a CFR of zero (other parameters matching influenza and Sars-Cov-2) and the maximum admitted contact rate for endemic equilibrium. C. Peak death rate at the height of an epidemic with contact rate corresponding to the maximum value admitting endemic equilibrium given $\beta=1$ and varying $\beta$ (decreasing $\beta$ corresponds to increasing isolation). Death rate is scaled by the maximum value for each virus. 


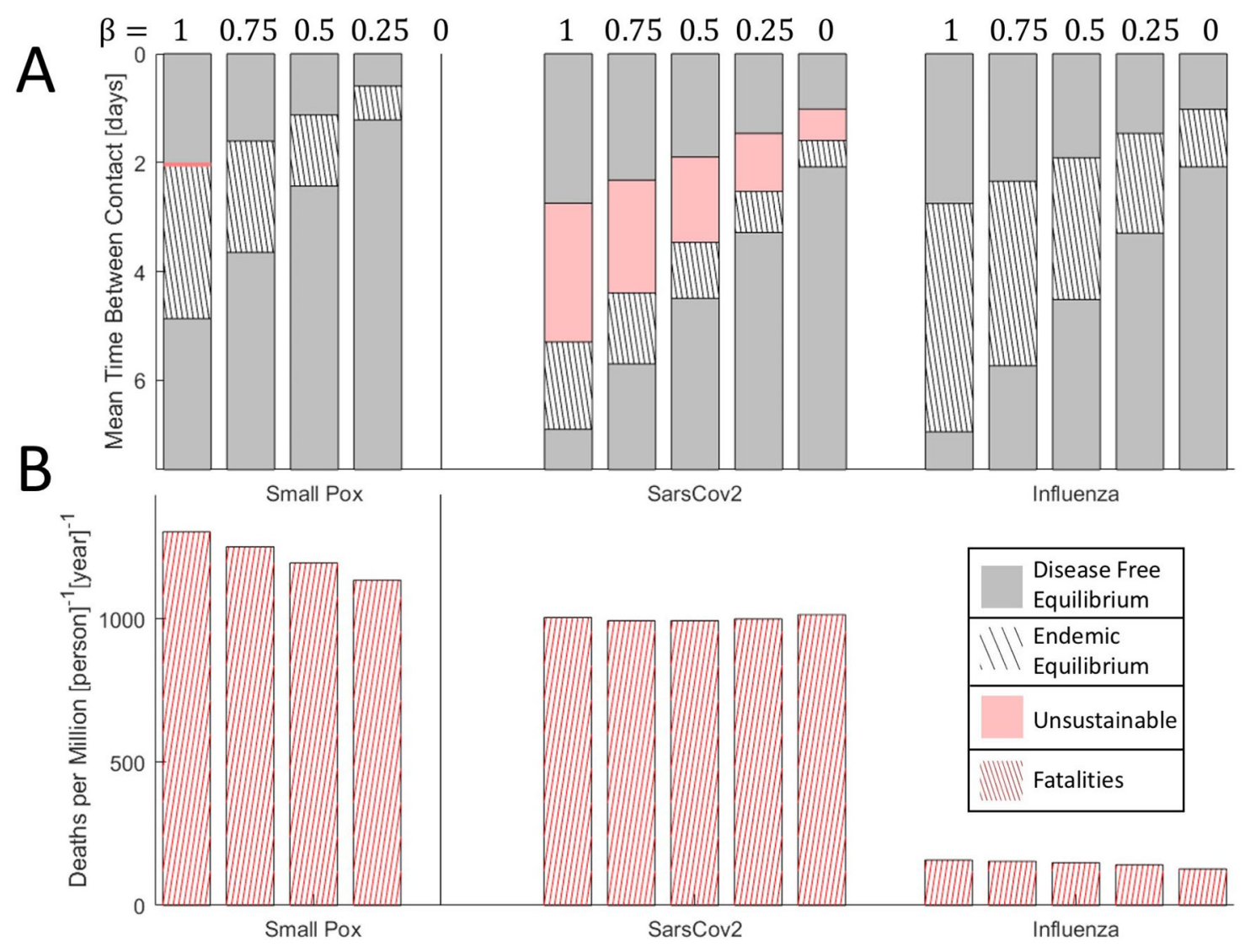

Figure 5. The effects of isolation on epidemic evolution. A. Disease free equilibrium (gray), endemic equilibrium (striped), and unsustainable (red) regions for three viruses over a range of host contact rates. Red region for smallpox-like viruses is narrow and not shown to scale. Bars are ordered by decreasing $\beta \in\{0,0.25,0.5,0.75,1\}$. B. Death rate at endemic equilibrium (contact rates correspond to the midrange shown in A). Bars are ordered by decreasing $\beta \in\{0,0.25,0.5,0.75,1\}$. Note that, representing equilibrium, the choice of timescale (years) is arbitrary.

host-host contact rate moves the population farther from this boundary in the phase space and alleviates this kinetic constraint. Therefore, even if host-host contact rates cannot be reduced enough to break endemic equilibrium by reaching $R_{0}<1$, it's possible that a modest reduction can change the evolutionary trajectory of the pathogen from one of stagnant or increasing CFR to one of decreasing CFR.

One way a host population can effectively decrease the rate of infection is through the isolation of symptomatic individuals. Isolation decreases death rate at the peak of the epidemic (Figure 4C) and can prevent an epidemic entirely by driving $R_{0}$ below 1 , which requires $\beta<\left(\frac{k_{R}+k_{D}+k_{P}}{k_{I}}-1\right)\left(\frac{k_{R}+k_{D}+k_{D V}}{k_{P}}\right)$. For pathogens with substantial asymptomatic or pre-symptomatic spread ( $\left(\right.$ mall $k_{P}$ ), this approach might not be feasible. Although isolation narrows the range of contact rates that admits endemic equilibria or unsustainability (Figure $5 \mathrm{~A}$ ), the death rate at endemic equilibrium varies little with decreasing $\beta$ and may even increase in the case of SARS-CoV-2-like viruses. Notably, however, SARS-CoV-2-like viruses are particularly sensitive to changes in $\beta$ such that a modest decrease in $\beta$, that is, increased isolation, can change the long-term outcome from endemic equilibrium to disease-free equilibrium. Furthermore, despite a 30-fold difference in CFR, both smallpox-like viruses and SARS-CoV-2-like viruses lead to the death of approximately $0.1 \%$ of the host population per year at endemic equilibrium. This highlights how pathogens with low or intermediate CFR can cause as many fatalities as a pathogen with a much higher CFR if allowed to reach endemic equilibrium.

\section{Discussion}

In this work, we analyzed the evolutionary regimes of human respiratory viruses with different life history traits. We did not conduct an invasion analysis, which would render many evolutionary paths within endemic equilibrium (Figure 3) inaccessible under specific conditions. Furthermore, we did not implement an explicit trade-off function. We consider any combination of life history characteristics that admit endemic equilibrium to be potentially biologically viable. 
Despite this maximally general and flexible approach, we demonstrate a highly constrained landscape for the evolution of human respiratory virus life-history traits and in particular show that CFR reduction is often constrained.

We draw an analogy between the evolution of pathogen life history traits and reversible chemical reactions where the "stability" of each set of life history traits is estimated by the total number of infected hosts at endemic equilibrium. The maximum size for the viral population and the minimum cost to the host is achieved at a CFR of 0 . This represents the globally stable state. In other words, the evolution of CFR is never thermodynamically constrained. In contrast, traversing evolutionary paths in the direction of this globally stable state often requires simultaneously modifying multiple parameters, some of which might be determined by host behavior, resulting in a high "activation energy". Therefore, the evolution of reduced CFR is often kinetically constrained. ${ }^{36}$

These constraints represent the most general limitations on potential evolutionary trajectories and likely play a key role in shaping the host-pathogen relationship for important human respiratory viruses. A reduction in CFR increases the average amount of time an infected host spends in the clinical compartment. This increases the total number of new infections that result from contact with each infected host. For smallpox-like pathogens with high CFR (the virulence/ infectivity trade-off) in communities with frequent contact, this can create a bottleneck in the size of the susceptible population, resulting in disease-free equilibrium (that is, extinction of the virus) when not accompanied by a decrease in the rate of infection. The rate of infection is determined by both the probability of infection given host-host contact and the host-host contact rate. Under these conditions, if the probability of infection following host contact were internally constrained by CFR, this would not constitute a fitness trade-off and could facilitate host adaptation. On the other hand, high host-host contact rates could externally constrain the rate of infection making reduction in CFR costly to the virus. Evolution of the smallpox virus shows a steady pattern of gene losses that likely lead to increasing infectivity and virulence. ${ }^{37,38}$ This evolutionary trend appears to be compatible with the conclusion that high CFR, smallpox-like viruses are unlikely to evolve towards decreasing CFR due to constraints imposed by the host behavior. Similar constraints have likely led to the emergence of acuteness for human bacterial respiratory pathogens of the genus Bordetella. ${ }^{18}$

For SARS-CoV-2-like pathogens with moderate CFR, evolution towards decreased CFR can be kinetically constrained by the relationship between CFR and the duration of immunity. Decreasing the duration of immunity increases the size of the infected population and the overall death rate which can make the host-pathogen relationship unsustainable. The existence of a large region of the phase space corresponding to unsustainable or kinetically constrained moderate-CFR viruses implies two distinct forms of host response over two different timescales. Over long timescales, unsustainable viruses are likely to face extinction due to the elimination of the susceptible host population. Although, in principle, this could occur via extinction of the entire host population, the emergence of host resistance is likely. Over short timescales, especially for modern human populations, the emergence of an unsustainable virus, such as SARS-CoV-2, may be considered societally unacceptable, leading to drastic measures that result in a major reduction in host-host contact rates. Both trends likely contribute to the paucity of moderate CFR human respiratory viruses which are subject to this kinetic constraint between immunity and CFR.

Perhaps paradoxically, immune evasion could incur a fitness cost for the pathogen and even lead to its extinction due to the host response. However, some level of immune evasion is required, among host populations with a low birthrate, to maintain any state of endemic equilibrium in the case where lifelong immunity is conferred against individual strains and the duration of immunity is determined by antigenic drift rather than the decline of immunity itself. Evolution towards decreased CFR is uncertain in this case, and a better understanding of internal genomic constraints ${ }^{7}$ could help predict the effects of immunomodulation. This is important when assessing the impact of novel or imperfect vaccination which can lead to counterintuitive results. ${ }^{15,39,40}$ Diversification related to immune evasion commonly enables the maintenance of large virus populations over long time scales, as is the case for influenza. ${ }^{41-43}$ In the case of SARS-CoV-2, immune evasion, diversification, and host adaptation of SARS-CoV-2 have already been demonstrated among a growing ensemble of variants bearing diverse mutations. ${ }^{44-46}$ Although these mutations are epidemiologically consequential on the timescale of the ongoing pandemic, it is important to note that there is no indication of any of these variants falling outside of the broad SARS-CoV-2-like parameter regime studied in this work. Furthermore, products of virus genes that are specifically found in pathogenic beta-coronaviruses have been implicated in immunomodulation, ${ }^{47}$ suggesting that the virus adapts to maintain an endemic equilibrium in this way. Although the present model cannot predict whether SARS-CoV-2 becomes more or less virulent through the course of our pandemic, our results strongly suggest that evolution of its CFR is kinetically constrained such that the region of the parameter space where reduced CFR could evolve is small. On the other hand, we show that even modestly decreasing the host-host contact rate can alleviate this kinetic constraint and promote CFR reduction. 
During both the ongoing SARS-CoV-2 pandemic and the first SARS-CoV-1 epidemic, stringent public health measures were taken to limit infection, extending beyond isolation of symptomatic individuals and into the quarantine of asymptomatic, and likely uninfected, contacts. ${ }^{22,31}$ Although only a crude depiction of the nuanced dynamics underlying SARS-CoV-2 infections, the analysis presented here suggests that isolation and quarantine are particularly effective towards changing the long-term outcome for viruses with moderate CFR and high probability of infection following host contact, such as SARS-CoV-2. The phase diagram for SARS-CoV-2-like viruses is sensitive to the parameter $\beta$ which reflects the isolation of symptomatic individuals (and additionally asymptomatic carriers who have tested positive). The evolution of the epidemic for such viruses is dominated by disease-free equilibrium or an unsustainable host-virus relationship. Endemic equilibrium is possible only in a narrow parameter range and is therefore unlikely. Nonetheless, the existence of this range suggests that herd immunity is unlikely to be achievable in the absence of vaccination, and if reached, would amount to an extreme number of fatalities. Our analysis shows that, while highly amenable to public health intervention, SARS-CoV-2-like viruses can be expected to contribute to a substantially higher death toll than influenza-like viruses, comparable instead to that of a smallpox-like virus, over a protracted period.

We emphasize one last time that our aim in this work was to use a maximally general model to chart the global phase space of human respiratory viruses. We did not include any explicit trade-off functions or specify within-host pathogen dynamics. Despite this maximally general and flexible approach, we demonstrate a highly constrained landscape for the evolution of human respiratory virus life-history characteristics and in particular show that CFR reduction is often constrained. Given the generality and simplicity of this approach, these results cannot be immediately leveraged to predict the outcome of specific epidemics for specific host populations. Such prediction requires an invasion analysis, taking into account many additional factors. ${ }^{5,17,18}$ Furthermore, throughout this work, we discuss ways in which host-pathogen interactions affect evolutionary outcomes. It is also important to acknowledge that environmental influences, perhaps most prominently the stability of the exposed pathogen, ${ }^{48}$ can additionally constrain the evolutionary outcome of an epidemic. Additionally, the model presented here is deterministic and assumes a large, well-mixed host population. Finite size effects for small or spatially segregated host populations can additionally affect epidemic outcome, ${ }^{2}$ resulting in substantial stochasticity.

\section{Conclusions}

Human respiratory virus epidemics often evolve under kinetic constraints such that the total number of infected hosts would increase if the CFR decreased, to the benefit of both the host and the pathogen. However, traversing the evolutionary paths to that state is unlikely, requiring simultaneous modification of multiple parameters, some of which are determined by the host behavior and jointly translate into a high "activation energy". These constraints can prevent the reduction in CFR for both high- and moderate-CFR viruses. The incorporation of these constraints can assist in the interpretation of classical model results for epidemics where some parameters, such as host-host contact rate, are unknown. We show that SARS-CoV-2-like viruses are unlikely to reach a state of endemic equilibrium; however, the potential for such equilibrium implies that herd immunity without vaccination may be impossible. At equilibrium, moderate-CFR viruses can cause as many fatalities as high-CFR viruses with both SARS-CoV-2-like viruses and smallpox-like viruses leading to death of about $0.1 \%$ of the population per year. However, even partial isolation of symptomatic individuals can have a major effect not only by reducing the number of fatalities in the short term but also by potentially changing the evolutionary trajectory of the virus towards reduced CFR. Such simple public health interventions can continue to dramatically decrease the forecasted cost of SARS-CoV-2 over both the short and long term.

\section{Author contributions}

Conceptualization and Formal Analysis: NDR, YIW, and EVK.

Writing - Original Draft Preparation: NDR and EVK.

Writing - Review \& Editing: NDR, YIW, and EVK.

\section{Acknowledgements}

The authors thank Koonin group members for helpful discussions.

\section{Data availability}

Extended data

Zenodo: Extended Data for 'Evolution of human respiratory virus epidemics' https://doi.org/10.5281/zenodo. $4818157 .^{28}$ 
The project contains the following underlying data:

- Appendix.pdf

- FigureS1.pdf

Data are available under the terms of the Creative Commons Attribution 4.0 International license (CC-BY 4.0).

1. Gandon S, Day T: The evolutionary epidemiology of vaccination. J R Soc Interface. 2007; 4(16): 803-17.

PubMed Abstract | Publisher Full Text | Free Full Text

2. Lion S, Gandon S: Spatial evolutionary epidemiology of spreading epidemics. Proc Biol Sci. 2016; 283(1841): 20161170. PubMed Abstract | Publisher Full Text | Free Full Text

3. Frank SA: Models of parasite virulence. Q Rev Biol. 1996; 71(1): 37-78.

PubMed Abstract | Publisher Full Text

4. Day T, Proulx SR: A general theory for the evolutionary dynamics of virulence. Am Nat. 2004; 163(4): E40-E63.

PubMed Abstract | Publisher Full Text

5. Alizon S, de de Roode JC, Michalakis Y: Multiple infections and the evolution of virulence. Ecol Lett. 2013; 16(4): 556-67.

PubMed Abstract | Publisher Full Text

6. Cressler CE, McLeod DV, Rozins C, et al.: The adaptive evolution of virulence: a review of theoretical predictions and empirical tests. Parasitol. 2016; 143(7): 915-30.

PubMed Abstract | Publisher Full Text | Free Full Text

7. Geoghegan JL, Holmes EC: The phylogenomics of evolving virus virulence. Nat Rev Genet. 2018; 19(12): 756-69.

PubMed Abstract | Publisher Full Text | Free Full Text

8. Alizon S, Hurford A, Mideo N, et al.: Virulence evolution and the trade-off hypothesis: history, current state of affairs and the future. J Evol Biol. 2009; 22(2): 245-59.

PubMed Abstract | Publisher Full Text

9. Anderson RM, May RM: Coevolution of hosts and parasites. Parasitol. 1982; 85(Pt 2): 411-26.

PubMed Abstract | Publisher Full Text

10. Day T: On the evolution of virulence and the relationship between various measures of mortality. Proc Biol Sci. 2002; 269(1498): 1317-23.

PubMed Abstract | Publisher Full Text | Free Full Text

11. Alizon S, Michalakis Y: Adaptive virulence evolution: the good old fitness-based approach. Trends Ecol Evol. 2015; 30(5): 248-54. PubMed Abstract | Publisher Full Text

12. Leggett $\mathrm{HC}$, Cornwallis $\mathrm{CK}$, Buckling $\mathrm{A}$, et al.: Growth rate, transmission mode and virulence in human pathogens. Philos Trans R Soc Lond B Biol Sci. 2017; 372(1719): 20160094. PubMed Abstract | Publisher Full Text | Free Full Text

13. Gandon S, Mackinnon MJ, Nee S, et al.: Imperfect vaccines and the evolution of pathogen virulence. Nature. 2001; 414(6865): 751-6.

PubMed Abstract | Publisher Full Text

14. Mackinnon MJ, Read AF: Virulence in malaria: an evolutionary viewpoint. Philos Trans R Soc Lond B Bio/ Sci. 2004; 359(1446): 965-86. PubMed Abstract | Publisher Full Text | Free Full Text

15. Soubeyrand $B$, Plotkin SA: Antitoxin vaccines and pathogen virulence. Nature. 2002; 417(6889): 609-10. Publisher Full Text

16. Gandon S, Mackinnon MJ, Nee S, et al.: Antitoxin vaccines and pathogen virulence. Nature. 2002; 417(6889): 610.

17. Mideo N, Alizon S, Day T: Linking within-and between-host dynamics in the evolutionary epidemiology of infectious diseases. Trends Ecol Evol. 2008; 23(9): 511-7. PubMed Abstract | Publisher Full Text

18. King AA, Shrestha S, Harvill ET, et al.: Evolution of acute infections and the invasion-persistence trade-off. Am Nat. 2009; 173(4): 446-55.

PubMed Abstract | Publisher Full Text | Free Full Text

19. Lau LL, Cowling BJ, Fang VJ, et al.: Viral shedding and clinical illness in naturally acquired influenza virus infections. J Infect Dis. 2010;
201(10): 1509-16.

PubMed Abstract | Publisher Full Text | Free Full Text

20. Bi Q, Wu Y, Mei S, et al.: Epidemiology and transmission of COVID19 in 391 cases and 1286 of their close contacts in Shenzhen, China: a retrospective cohort study. Lancet Infect Dis. 2020; 20(8): 911-9.

PubMed Abstract | Publisher Full Text | Free Full Text

21. Lau EH, Hsiung CA, Cowling BJ, et al.: A comparative epidemiologic analysis of SARS in Hong Kong, Beijing and Taiwan. BMC Infect Dis. 2010; 10(1): 1-9.

PubMed Abstract | Publisher Full Text | Free Full Text

22. Barbisch D, Koenig KL, Shih F-Y: Is there a case for quarantine? Perspectives from SARS to Ebola. Disaster Med Public Health Prep. 2015; 9(5): 547-53.

PubMed Abstract | Publisher Full Text

23. Jacobs SE, Lamson DM, George KS, et al.: Human rhinoviruses. Clin Microbiol Rev. 2013; 26(1): 135-62. PubMed Abstract | Publisher Full Text | Free Full Text

24. Munywoki PK, Koech DC, Agoti CN, et al.: Frequent asymptomatic respiratory syncytial virus infections during an epidemic in a rural Kenyan household cohort.J Infect Dis. 2015; 212(11): 1711-8. PubMed Abstract | Publisher Full Text | Free Full Text

25. CDC. Reference Source

26. UpToDate. Reference Source

27. Y-j S, Yang J-S, Yoon HJ, et al.: Asymptomatic Middle East Respiratory Syndrome coronavirus infection using a serologic survey in Korea. Epidemiol Health. 2018; 40. PubMed Abstract | Publisher Full Text | Free Full Text

28. Rochman ND, Wolf YI, Koonin EV Evolution of human respiratory virus epidemics: Extended data. Zenodo. 2021. PubMed Abstract | Publisher Full Text | Free Full Text

29. Arino J, Brauer $F$, van den Driessche $P$, et al.: Simple models for containment of a pandemic. J $R$ Soc Interface. 2006; 3(8): 453-7. PubMed Abstract | Publisher Full Text | Free Full Text

30. Brauer F: Some simple epidemic models. Math Biosci Eng. 2006; 3(1): 1 .

PubMed Abstract | Publisher Full Text

31. Lipsitch M, Cohen T, Cooper B, et al.: Transmission dynamics and control of severe acute respiratory syndrome. Science. 2003; 300(5627): 1966-70.

PubMed Abstract | Publisher Full Text | Free Full Text

32. Diekmann $\mathrm{O}$, Heesterbeek J, Roberts MG: The construction of nextgeneration matrices for compartmental epidemic models. $J R$ Soc Interface. 2010; 7(47): 873-85.

PubMed Abstract | Publisher Full Text | Free Full Text

33. Guo H, Li MY, Shuai Z: Global stability of the endemic equilibrium of multigroup SIR epidemic models. Can Appl Math Q. 2006; 14(3): 259-84.

Publisher Full Text

34. Sun R: Global stability of the endemic equilibrium of multigroup SIR models with nonlinear incidence. Comput Math Appl. 2010; 60(8): 2286-91. Publisher Full Text

35. Toolbox SM: Matlab. Mathworks Inc. 1993

36. Lion S, Metz JA: Beyond RO maximisation: on pathogen evolution and environmental dimensions. Trends Ecol Evol. 2018; 33(6): 458-73.

PubMed Abstract | Publisher Full Text

37. Mühlemann B, Vinner L, Margaryan A, et al.: Diverse variola virus (smallpox) strains were widespread in northern Europe in the 
Viking Age. Science. 2020; 369(6502)

PubMed Abstract | Publisher Full Text

38. Hendrickson RC, Wang C, Hatcher EL, et al.: Orthopoxvirus genome evolution: the role of gene loss. Viruses. 2010; 2(9): 1933-67. PubMed Abstract | Publisher Full Text | Free Full Text

39. Vale PF, Fenton A, Brown SP: Limiting damage during infection lessons from infection tolerance for novel therapeutics. PLOS Biol. 2014; 12(1): e1001769.

PubMed Abstract | Publisher Full Text | Free Full Text

40. Rochman ND, Wolf YI, Koonin EV: Substantial Impact of Post Vaccination Contacts on Cumulative Infections during Viral Epidemics. medRxiv. 2020.

Publisher Full Text

41. Wolf YI, Viboud C, Holmes EC, et al.: Long intervals of stasis punctuated by bursts of positive selection in the seasonal evolution of influenza A virus. Biol Direct. 2006; 1(1): $1-19$.

PubMed Abstract | Publisher Full Text | Free Full Text

42. Gong LI, Suchard MA, Bloom JD: Stability-mediated epistasis constrains the evolution of an influenza protein. Elife. 2013; 2: e00631.

PubMed Abstract | Publisher Full Text | Free Full Text
43. Kryazhimskiy S, Dushoff J, Bazykin GA, et al.: Prevalence of epistasis in the evolution of influenza A surface proteins. PLoS Genet. 2011; 7(2): e1001301.

PubMed Abstract | Publisher Full Text | Free Full Text

44. Rochman ND, Wolf YI, Faure G, et al.: Ongoing Global and Regional Adaptive Evolution of SARS-CoV-2. bioRxiv. 2020. Publisher Full Text

45. Zhang $Y$, Zhang J, Chen $Y$, et al.: The ORF8 protein of SARS-CoV-2 mediates immune evasion through potently downregulating MHC-I. bioRxiv. 2020.

46. Korber B, Fischer WM, Gnanakaran S, et al.: Tracking changes in SARS-CoV-2 Spike: evidence that D614G increases infectivity of the COVID-19 virus. Cell. 2020; 182(4): 812-27. e19. PubMed Abstract | Publisher Full Text | Free Full Text

47. Tan $Y$, Schneider $T$, Leong $M$, et al.: Novel immunoglobulin domain proteins provide insights into evolution and pathogenesis of SARS-CoV-2-related viruses. MBio. 2020; 11(3). PubMed Abstract | Publisher Full Text | Free Full Text

48. Walther BA, Ewald PW: Pathogen survival in the external environment and the evolution of virulence. Biol Rev. 2004; 79(4): 849-69.

PubMed Abstract | Publisher Full Text | Free Full Text 


\section{Open Peer Review}

\section{Current Peer Review Status:}

\section{Version 2}

Reviewer Report 20 August 2021

https://doi.org/10.5256/f1000research.58799.r90715

(c) 2021 Sneppen K. This is an open access peer review report distributed under the terms of the Creative Commons Attribution License, which permits unrestricted use, distribution, and reproduction in any medium, provided the original work is properly cited.

\section{Kim Sneppen}

Niels Bohr Institute, University of Copenhagen, Copenhagen, Denmark

I am happy with the clarifications provided by the authors.

As minor comments, I still found the picture of a rate being proportional to activation energy $E$ strange, since rates are rather proportional to exp(-E/Temperature). Also the following paragraph implying an detailed balance for diseases, somewhat misrepresenting SIRS, i.e. a cyclic set of reactions where there is no detailed balance (but of course a steady endemic state). The authors probably work on a more philosophical level here, but that does not make the analogy clearer.

Competing Interests: No competing interests were disclosed.

\section{I confirm that I have read this submission and believe that I have an appropriate level of expertise to confirm that it is of an acceptable scientific standard.}

Reviewer Report 10 August 2021

\section{https://doi.org/10.5256/f1000research.58799.r90716}

(C) 2021 Wilke C. This is an open access peer review report distributed under the terms of the Creative Commons Attribution License, which permits unrestricted use, distribution, and reproduction in any medium, provided the original work is properly cited.

\section{Claus O. Wilke}

Department of Integrative Biology, The University of Texas, Austin, TX, USA

The article is very much improved after revision and I have no further comments or concerns.

Competing Interests: No competing interests were disclosed. 
Reviewer Expertise: mathematical modeling, evolutionary biology, infectious disease

I confirm that I have read this submission and believe that I have an appropriate level of expertise to confirm that it is of an acceptable scientific standard.

\section{Version 1}

Reviewer Report 07 July 2021

https://doi.org/10.5256/f1000research.56772.r86917

(C) 2021 Sneppen K. This is an open access peer review report distributed under the terms of the Creative Commons Attribution License, which permits unrestricted use, distribution, and reproduction in any medium, provided the original work is properly cited.

\section{Kim Sneppen}

Niels Bohr Institute, University of Copenhagen, Copenhagen, Denmark

The paper investigates the sustainability of steady state (endemic states) of various diseases. Given the paper is a rather simple modification of a SIRS model, the paper is a remarkable difficult read. Thus I may easily be missing something due to my difficulty in following the logic.

1. The title mention evolution, but I failed to find what is evolving, probably because I could not grasp the true meaning of the kinematic constraints, i.e. what mechanism among the virus should want to drive it towards a steady state endemic state, given that the virus tend to be selected to maximize its offspring from generation to generation? A more clear explanation of this potentially important new scale of evolutionary selection would be helpful.

2. Overall, I am uncertain about the steady state approach to the diseases, as endemic like diseases often comes in pulses, like smallpox in the late 18th century, or perhaps like standard influenza epidemics (with all their caveats on mutations and partial crossimmunity).

3. In more detail, I am sorry, but do not get the logic of Fig. 1. Clearly, a disease can eventually go extinct because either the transmission rate is to low, or so high that one fast reach a state where herd immunity makes further propagation impossible. In between these extremes there will be some endemic state, supported by susceptible from birth or from loss of immunity from previously infected people.

4. Table 2 does not give a hint about real differences in infection rates between smallpox and coronavirus. Apart from that then the parameters from SARS-CoV-2 is misleading, as serial interval and infectious period each is about 5 days.

5. Fig. 2 nicely outlines the model, but then in panel $B, C)$ presents data using a $y$-axis that quantifies $\underline{\mathrm{kI}}($ ?) in contact days? What about infectivity, some diseases like smallpox have a R0 of about 20, while other like SARS have R0 between 2 and 5. How does the y-axis in Fig. 2 
relate to these very different scenarios?

6. Fig. 3 is again a hard figure to read? How can smallpox softly transit from SARS-Cov-2. I do not get how increased case fatality rate changes the regime of the sustainable endemic state? Also, the disease free equilibrium at short contact times presumably reflects situations where the first epidemic was so brutal that herd immunity was more than obtained? I don't understand the unsustainable red region? Why larger for larger CFR?

7. Fig. 4 is difficult given the arbitrariness of all parameters. What is the point of 3 sub-figures, what could I as a reader learn?

8. Fig. 5 investigates mitigation, something that has little to do with endemic states (presumably we would not like to have a permanently mitigated society?). I also failed to locate whether the mitigation fraction beta only included reduction in the symptomatic transmission? For SARS-CoV-2 there is a lot of asymptomatic transmission, but not for SARS.

9. In conclusion, the authors say that SARS-CoV-2 cannot reach a state of endemic equilibrium. They might be right, but one might as well imagine that disease immunity decline sufficiently fast that it could be constantly circulating in some mild form. Also, I cannot see how its increasingly many mutant variants would make such a conclusion robust.

10. In the results section of the abstract the authors state: "We reveal kinetic constraints where the variation of multiple parameters in concert leads to decreased CFR and increased pathogen fitness, whereas independent variation of the parameters decreases pathogen fitness. Smallpox, SARS-CoV-2, and influenza are analyzed as diverse representatives of human respiratory viruses. We show that highly virulent viruses, such as smallpox, are likely often constrained by host behavior, whereas moderately virulent viruses, such as SARS-CoV2 , appear to be typically constrained by the relationship between the duration of immunity and CFR." This is beyond my understanding: smallpox could not really be constrained (or could not when it recurrently broke out in cities in the 18th century). SARS-CoV-2 was in contrast contained by human mitigation, and had little directly to do with CFR.

11. A minor point is the strange discussion of reversible chemical reactions in a paragraph on page 2 . It seems to assume that energy is directly proportional to transmission rates. Also its relation to the rest of the manuscript is unclear.

12. Overall, the paper presents a worthwhile investigation into an important phenomenon. However, it is very difficult to read and evaluate given its unclear assumptions (and complicated sentences). I would encourage a major rewrite, emphasizing which few (hopefully) parameters that are important, and with new figures that more clearly illustrate the main point.

\section{Is the work clearly and accurately presented and does it cite the current literature?} Partly

Is the study design appropriate and is the work technically sound?

Partly 
Are sufficient details of methods and analysis provided to allow replication by others? Partly

If applicable, is the statistical analysis and its interpretation appropriate?

Not applicable

Are all the source data underlying the results available to ensure full reproducibility? Partly

Are the conclusions drawn adequately supported by the results?

No

Competing Interests: No competing interests were disclosed.

I confirm that I have read this submission and believe that I have an appropriate level of expertise to state that I do not consider it to be of an acceptable scientific standard, for reasons outlined above.

Author Response ( F1000Research Advisory Board Member ) 17 Jul 2021

Eugene Koonin, National Institutes of Health, Bethesda, USA

Response to Kim Sneppen:

The paper investigates the sustainability of steady state (endemic states) of various diseases. Given the paper is a rather simple modification of a SIRS model, the paper is a remarkable difficult read. Thus I may easily be missing something due to my difficulty in following the logic.

Response: We apologize for any confusion and hope that our revised presentation is substantially clearer.

The title mention evolution, but I failed to find what is evolving, probably because I could not grasp the true meaning of the kinematic constraints, i.e. what mechanism among the virus should want to drive it towards a steady state endemic state, given that the virus tend to be selected to maximize its offspring from generation to generation? A more clear explanation of this potentially important new scale of evolutionary selection would be helpful. Overall, I am uncertain about the steady state approach to the diseases, as endemic like diseases often comes in pulses, like smallpox in the late 18th century, or perhaps like standard influenza epidemics (with all their caveats on mutations and partial cross-immunity).

Response: This study is primarily concerned with very long-term evolutionary outcomes. The reviewer makes the important point, which has been emphasized in the revised version that, in reality, a pathogen rarely reaches a true steady state. Outbreaks are almost always spatially and/or seasonally dependent, and the fraction of a host population that is infected fluctuates significantly over time. However, even diseases like Influenza or Smallpox that are or were subject to periodic epidemics or pandemics, during which the number of infected individuals is much higher than average, both are or were endemic to the human population such that a predictable number of cases would be sustained on average within a year or a decade. This study 
largely focuses on such averages as emphasized in the revision.

In more detail, I am sorry, but do not get the logic of Fig. 1. Clearly, a disease can eventually go extinct because either the transmission rate is to low, or so high that one fast reach a state where herd immunity makes further propagation impossible. In between these extremes there will be some endemic state, supported by susceptible from birth or from loss of immunity from previously infected people.

Response: Figure 1 was introduced to provide context for a broad audience. The reviewer is correct that the content of this figure may be obvious for an expert reader. Indeed, the logic is described correctly in the reviewer's comment. We still think that this figure is useful for a reader unfamiliar with specifics of evolutionary epidemiology, and under the current circumstances, we expect there will be many such readers of this paper.

Table 2 does not give a hint about real differences in infection rates between smallpox and coronavirus. Apart from that then the parameters from SARS-CoV-2 is misleading, as serial interval and infectious period each is about 5 days.

Response: The reviewer is correct that Table 2 does not list the differences in the rates of infection. This is because the rate of infection is the triple product of the rate of contact between hosts, the fraction of the population (not isolated) which is infected, and the probability of infection upon contact. Notwithstanding the variability in the probability of infection upon contact, which itself depends on the viral load of the infected individual and the nature of contact, substantial variability of the rate of contact between hosts makes it extremely challenging to report globally meaningful rates of infection for pathogens, and especially, respiratory pathogens. We agree that this table could be misleading considering the large values reported for the time symptomatic, which does not reflect the fact that hosts may be symptomatic but no longer infectious. This has been clarified in the text. SARS-CoV-2 is modelled with a 7 day period, during which the host is infectious and symptomatic, preceded by a 3 day asymptomatic and infectious period. This was the consensus prediction at the time this manuscript was initially prepared and we have acknowledged in the revised text that, given its recent identification, the best fit parameter values for SARS-CoV-2 are subject to change. We do not model the serial interval because, like the rate of infection, it depends on the rate of contact.

Fig. 2 nicely outlines the model, but then in panel $B, C)$ presents data using a $y$-axis that quantifies $\mathrm{kI}(?)$ in contact days? What about infectivity, some diseases like smallpox have a RO of about 20, while other like SARS have RO between 2 and 5. How does the y-axis in Fig. 2 relate to these very different scenarios?

Response: We thank the reviewer for their appreciation of this figure and regret any possible confusion. As discussed in the previous comment, the rate of infection is the triple product of the rate of contact between hosts, the fraction of the population (not isolated) which is infected, and the probability of infection upon contact. kI, as listed in Table 1, is the product of the rate of contact and the probability of infection upon contact: kcontact*P (infect). In principle, both may vary, so for simplicity, we study this compound parameter, which can increase as a result of either increasing P(infect) or increasing kcontact. As $\mathrm{P}$ (infect) is unitless, $\mathrm{kI}$ has units of $1 /$ time and varying $\mathrm{kI}$ is equivalent to varying the time between contact, which is what we report. While this was stated in the original version where the model is initially described, we acknowledge the presentation could use improvement and apologize for any 
confusion. We have added additional clarification on the nature of $\mathrm{kI}$ and the units of the $y$-axis in the figures. Additionally, we note that R0 depends on the rate of host contact and so will also vary across communities and over time for a single pathogen. Fig. 3 is again a hard figure to read? How can smallpox softly transit from SARS-Cov-2. I do not get how increased case fatality rate changes the regime of the sustainable endemic state? Also, the disease free equilibrium at short contact times presumably reflects situations where the first epidemic was so brutal that herd immunity was more than obtained? I don't understand the unsustainable red region? Why larger for larger CFR? Response: We regret the confusion. The reviewer is completely correct that smallpox cannot smoothly transit from SARS-CoV-2. This is evident in Figure 3 because there is no path from the smallpox-like regime to the SARS-CoV-2-like regime that does not exit endemic equilibrium. The solid line in the plot indicates that to the right of the line, the pathogen confers permanent immunity and to the left of the line, temporary immunity. The dashed line represents a smooth transition between the SARS-CoV-2like regime and the Influenza-like regime, which are both modelled to produce temporary immunity. This has been clarified in the revised version. The duration of immunity is the third key parameter that is varied in Figure 4A. Increased CFR changes the regime of the sustainable endemic state because it changes the balance between births and deaths in the population. An endemic pathogen with a high CFR results in a greater number of mortalities per year for the host population. The reviewer is correct that disease-free equilibrium at short contact times reflects a selective bottleneck in the host population as illustrated in Figure 1B. This has been emphasized in the revised version. However, it should be noted that a selective bottleneck at low CFR does not necessarily represent an outbreak resulting in significant mortality. The unsustainable red region represents the case where endemic equilibrium is possible but eventually, because the number of deaths sustained by the pathogen exceeds the birth rate, the host population will either go extinct or must adapt in some way to avoid the pathogen. This represents an unsustainable host-pathogen relationship. This region is larger for larger CFR because the number of deaths sustained by the population increases with increasing CFR.

Fig. 4 is difficult given the arbitrariness of all parameters. What is the point of 3 subfigures, what could I as a reader learn?

Response: We respectfully suggest that the parameters are not arbitrary. Figures 3 and $4 \mathrm{~A}$ are critical to understanding the life history phase space that is available human respiratory viruses. Our choice of parameter exploration in the initial submission was motivated by the range of parameters observed for pathogenic human respiratory viruses. Additionally, where possible, analytic dependencies on the size of the clinical population at endemic equilibrium are presented in the Appendix. These pathogens span a wide range of CFR, and host-host contact rates can vary significantly among human populations. We additionally vary the amount of time a host is both asymptomatic and infectious although we do not allow this parameter to vary freely because that would have been implausible from a biological standpoint: hosts infected with high CFR viruses are not known to spend significant time in an asymptomatic and infectious state. We additionally explore the full range of immune duration (parametrized by $0<=$ alpha $<=1$ ) for SARS-CoV-2-like and Influenza-like viruses. This parameter largely determines the region of the phase 
space that corresponds to unsustainability, and representative values are shown in red curves in figure $4 \mathrm{~A}$. This covers the CFR (k_DV), the rate of progression (k_P), the rate of contact/probability of infection (k_I), and the rate at which immunity is lost $\left(k_{-} L\right)$. We discuss the importance of small birth rate, and a small difference between k_B and k_D (deathrate not specific to the pathogen), for interpreting our results and linearization of the model requires that value to be small. These rates are expected to vary significantly less among human populations than pathogen-specific rates and we submit are not critical to vary. Lastly, we devote the final figure to studying the effects of the parameter beta representing isolation of symptomatic individuals. Thus we hope we have demonstrated to the reviewer that we effectively surveyed the entire parameter space admitting endemic equilibrium for the pathogens of interest. Additionally, although we continue to maintain that the parameters fully explored in the main text constitute those of greatest relevance given the known variability among human respiratory viruses, we acknowledge that a demonstration of the dependence on $\mathrm{k} \_\mathrm{R}$ is useful for the reader as well. This is available as a supplementary figure. We agree that panels $B$ and $C$ are perhaps less critical to understanding the key results of this study, but still contain information central to what is discussed in the text. Specifically, panel B demonstrates that the size of the viral population at endemic equilibrium decreases with an increasing duration of immunity. Panel $\mathrm{C}$ demonstrates the reduced cost of an epidemic resulting in reducing the contact rate among symptomatic hosts and is a segue into the final figure.

Fig. 5 investigates mitigation, something that has little to do with endemic states (presumably we would not like to have a permanently mitigated society?). I also failed to locate whether the mitigation fraction beta only included reduction in the symptomatic transmission? For SARS-CoV-2 there is a lot of asymptomatic transmission, but not for SARS.

Response: We regret any confusion. Mitigation may refer to either the isolation of symptomatic individuals or the quarantine of asymptomatic, and likely uninfected, contacts. While we discussed this in the original version, this has been emphasized in the revised version. We only model the former - isolation of symptomatic hosts and would argue that in that sense we already live in a "permanently mitigated society". Such mitigation has a dramatic effect on the range of host-host contact rates which admit endemic equilibrium as displayed in Figure 5A. The reviewer is correct that the death rate at endemic equilibrium is insensitive to mitigation. This is displayed in Figure 5B. The reviewer is also correct that asymptomatic transmission for SARS-CoV2 is more substantial than it was for SARS. We discuss how high CFR pathogens are generally subject to less asymptomatic transmission than those with lower CFR. In conclusion, the authors say that SARS-CoV-2 cannot reach a state of endemic equilibrium. They might be right, but one might as well imagine that disease immunity decline sufficiently fast that it could be constantly circulating in some mild form. Also, I cannot see how its increasingly many mutant variants would make such a conclusion robust.

Response: We agree that the wording in the abstract was somewhat overstated, so we have amended it in the revision. In the main text we state, "We show that SARSCoV-2-like viruses are unlikely to reach a state of endemic equilibrium; however, the potential for such equilibrium implies that herd immunity without vaccination might 
not be achievable". We do not intend to state SARS-CoV-2 "cannot" reach a state of endemic equilibrium. The reviewer suggests that the duration of immunity may decline sufficiently fast so that the pathogen could circulate in some mild form. This implicitly assumes that, in addition to a reduction in the duration of immunity, SARSCoV-2 would also evolve towards a reduction in CFR. This is precisely what we discuss. Regarding the emergence of novel variants, we think it is important to emphasize that antigenic drift is a major driver of endemic equilibrium, and furthermore, that all SARS-CoV-2 variants still firmly reside in the SARS-CoV-2-like region of the global phase space. We have clarified this point in the revised text.

In the results section of the abstract the authors state: "We reveal kinetic constraints where the variation of multiple parameters in concert leads to decreased CFR and increased pathogen fitness, whereas independent variation of the parameters decreases pathogen fitness. Smallpox, SARS-CoV-2, and influenza are analyzed as diverse representatives of human respiratory viruses. We show that highly virulent viruses, such as smallpox, are likely often constrained by host behavior, whereas moderately virulent viruses, such as SARS-CoV-2, appear to be typically constrained by the relationship between the duration of immunity and CFR." This is beyond my understanding: smallpox could not really be constrained (or could not when it recurrently broke out in cities in the 18th century). SARSCoV-2 was in contrast contained by human mitigation, and had little directly to do with CFR.

Response: We regret any confusion. In this study, we discuss both evolutionary constraints as well as the reduction in the rate of contact for symptomatic hosts, containment. We demonstrate that the evolution of smallpox-like viruses is likely constrained so that the CFR of smallpox remained high throughout the entire period over which it was endemic to human populations - well in line with the reviewer's comments. We do not suggest that smallpox was historically contained; however, we do demonstrate that due to the relatively few asymptomatic transmission events associated with smallpox, in principle, containment may be possible for a modern society. Furthermore, we would argue that the CFR of SARS-CoV-2 played a key role in its containment. Should a novel virus have emerged with a much lower CFR, containment strategies would likely not have been pursued at all.

A minor point is the strange discussion of reversible chemical reactions in a paragraph on page 2. It seems to assume that energy is directly proportional to transmission rates. Also its relation to the rest of the manuscript is unclear.

Response: This discussion serves to introduce the concepts of kinetic and thermodynamic constraints to endemic equilibrium analogous to kinetic and thermodynamically limited chemical reactions. We believe that this analogy is illustrative of the evolutionary trajectories of viruses on multidimensional fitness landscapes and include this discussion in an effort to make the study more approachable to a broad audience. As is the case with Figure 1, we acknowledge that an expert reviewer may find this discussion unnecessary or perhaps even distracting. We apologize for any confusion and have further expanded the explanation of the idea of kinetic vs. thermodynamic constraints in the revised version in an effort to better connect this illustration to the topic at hand.

Overall, the paper presents a worthwhile investigation into an important phenomenon. However, it is very difficult to read and evaluate given its unclear assumptions (and complicated sentences). I would encourage a major rewrite, emphasizing which few 
(hopefully) parameters that are important, and with new figures that more clearly illustrate the main point.

Response: We thank the reviewer for their comments. We have substantially revised and amended the text in an effort to clarify the presentation; however, we have elected to maintain the original figures.

Competing Interests: I declare no competing interests.

Reviewer Report 25 June 2021

https://doi.org/10.5256/f1000research.56772.r87356

(C) 2021 Wilke C. This is an open access peer review report distributed under the terms of the Creative Commons Attribution License, which permits unrestricted use, distribution, and reproduction in any medium, provided the original work is properly cited.

\section{Claus O. Wilke}

Department of Integrative Biology, The University of Texas, Austin, TX, USA

Overall, this contribution is very well written and seems reasonable. I have no major concerns, but I do have a few comments:

1. The authors write "as well as the difficulty of vaccination against lower-CFR viruses, such as influenza, relative to higher-CFR viruses, such as smallpox". I don't believe that difficulty of vaccination is directly linked to CFR, except maybe in the sense that there's less economic incentive to develop a vaccine if CFR is low.

2. Table 1 looks a bit weird. Maybe use two columns?

3. While the paper is overall well written, in the end I find it difficult to figure out what exactly the authors' conclusion is. I think the problem lies in the term "kinetically constrained", which is used throughout the manuscript but never actually explained. In the end, evolution of CFR comes down to the existence (or lack thereof) of transmission-mortality trade-offs, and I think you're trying to say that these trade-offs can be shaped by the rate constants of the various "reactions" in your model. If so, can you say this explicitly? And also, would this be at all surprising? Can you make any stronger or more specific statements?

You may find this paper helpful as a demonstration of how one can talk about this topic in very clear and understandable terms (Bull et al. $\left(2014^{1}\right)$ ).

\section{References}

1. Bull JJ, Lauring AS: Theory and empiricism in virulence evolution.PLoS Pathog. 2014; 10 (10): e1004387 PubMed Abstract | Publisher Full Text 
Is the work clearly and accurately presented and does it cite the current literature? Yes

Is the study design appropriate and is the work technically sound?

Yes

Are sufficient details of methods and analysis provided to allow replication by others? Yes

If applicable, is the statistical analysis and its interpretation appropriate? Yes

Are all the source data underlying the results available to ensure full reproducibility? No source data required

Are the conclusions drawn adequately supported by the results?

Yes

Competing Interests: No competing interests were disclosed.

Reviewer Expertise: mathematical modeling, evolutionary biology, infectious disease

I confirm that I have read this submission and believe that I have an appropriate level of expertise to confirm that it is of an acceptable scientific standard, however I have significant reservations, as outlined above.

Author Response ( F1000Research Advisory Board Member ) 17 Jul 2021

Eugene Koonin, National Institutes of Health, Bethesda, USA

Response to Claus Wilke:

Overall, this contribution is very well written and seems reasonable. I have no major concerns, but I do have a few comments.

Response: We thank the reviewer for their kind remarks.

The authors write "as well as the difficulty of vaccination against lower-CFR viruses, such as influenza, relative to higher-CFR viruses, such as smallpox". I don't believe that difficulty of vaccination is directly linked to CFR, except maybe in the sense that there's less economic incentive to develop a vaccine if CFR is low.

Response: We agree with the reviewer that economic incentive is an important consideration which we did not mention in the previous version and have added in the revision. With this sentence, however, we intended to highlight the fact that lower CFR viruses are less likely to provide permanent immunity which in turn complicates vaccination strategies. This has been clarified in the revision.

Table 1 looks a bit weird. Maybe use two columns?

Response: We apologize if the former layout reduced the legibility of Table 1 . We have reformatted the table as suggested. 
While the paper is overall well written, in the end I find it difficult to figure out what exactly the authors' conclusion is. I think the problem lies in the term "kinetically constrained", which is used throughout the manuscript but never actually explained. In the end, evolution of CFR comes down to the existence (or lack thereof) of transmission-mortality trade-offs, and I think you're trying to say that these trade-offs can be shaped by the rate constants of the various "reactions" in your model. If so, can you say this explicitly? And also, would this be at all surprising? Can you make any stronger or more specific statements?

Response: We regret the confusion and have further expanded our discussion of kinetic vs. thermodynamic constraints in the revised version. Regarding the point that we are, "trying to say that these trade-offs can be shaped by the rate constants", this is certainly the case, and we agree with the reviewer that this is to be expected. In this work, however, we highlight the opposite. There are very broad trade-offs (or in some instances the lack of trade-offs), which are largely independent of model parameters and shared across broad regimes within the life history characteristic phase space of all human respiratory viruses. We infer these very general evolutionary constraints without the use of an explicit trade-off function and without specifying an intra-host evolutionary model. This results in a "10,000 foot view" of the landscape, very general but not highly precise. In the original manuscript, we were cautious not to overstate the predictive value of our approach for specific epidemic outcomes. We thank the reviewer for this comment and acknowledge that these efforts may have resulted in unnecessary ambiguity in the text. We have strengthened and reiterated the statement of the principal conclusions throughout the revised version.

You may find this paper helpful as a demonstration of how one can talk about this topic in very clear and understandable terms (Bull et al. (2014)).

Response: We thank the reviewer for bringing this work to our attention. We agree that it is highly relevant, most interesting and very clearly written. We have appropriately cited this work.

Competing Interests: I declare no competing interests. 
The benefits of publishing with F1000Research:

- Your article is published within days, with no editorial bias

- You can publish traditional articles, null/negative results, case reports, data notes and more

- The peer review process is transparent and collaborative

- Your article is indexed in PubMed after passing peer review

- Dedicated customer support at every stage

For pre-submission enquiries, contact research@f1000.com 